\title{
Global estimates for quasilinear parabolic equations on Reifenberg flat domains and its applications to Riccati type parabolic equations with distributional data
}

\author{
Quoc-Hung Nguyen ${ }^{1}$
}

Received: 23 November 2014 / Accepted: 14 September 2015 / Published online: 16 October 2015

(C) Springer-Verlag Berlin Heidelberg 2015

Abstract In this paper, we prove global weighted Lorentz and Lorentz-Morrey estimates for gradients of solutions to the quasilinear parabolic equations:

$$
u_{t}-\operatorname{div}(A(x, t, \nabla u))=\operatorname{div}(F),
$$

in a bounded domain $\Omega \times(0, T) \subset \mathbb{R}^{N+1}$, under minimal regularity assumptions on the boundary of domain and on nonlinearity $A$. Then results yields existence of a solution to the Riccati type parabolic equations:

$$
u_{t}-\operatorname{div}(A(x, t, \nabla u))=|\nabla u|^{q}+\operatorname{div}(F)+\mu,
$$

where $q>1$ and $\mu$ is a bounded Radon measure.

Mathematics Subject Classification Primary 35K59 - Secondary 42B37

\section{Introduction and main results}

In this article, we are concerned with the global weighted Lorentz space estimates for gradients of weak solutions to quasilinear parabolic equations in divergence form:

$$
\left\{\begin{array}{l}
u_{t}-\operatorname{div}(A(x, t, \nabla u))=\operatorname{div}(F) \text { in } \Omega_{T}, \\
u=0 \text { on } \partial_{p}(\Omega \times(0, T)),
\end{array}\right.
$$

where $\Omega_{T}:=\Omega \times(0, T)$ is a bounded open subset of $\mathbb{R}^{N+1}, N \geq 2, \partial_{p}(\Omega \times(0, T))=$ $(\partial \Omega \times(0, T)) \cup(\Omega \times\{t=0\}), F \in L^{1}\left(\Omega_{T}, \mathbb{R}^{N}\right)$ is a given vector field and the nonlinearity

Communicated by N. Trudinger.

Quoc-Hung Nguyen

Hung.Nguyen-Quoc@1mpt.univ-tours.fr; quoc-hung.nguyen@epfl.ch

1 École Polytechnique Fédérale de Lausanne, SB MATHAA CAMA, Station 8, 1015 Lausanne, Switzerland 
$A: \mathbb{R}^{N} \times \mathbb{R} \times \mathbb{R}^{N} \rightarrow \mathbb{R}^{N}$ is a Carathéodory vector valued function, i.e. $A$ is measurable in $(x, t)$ and continuous with respect to $\nabla u$ for a.e. $(x, t)$. We suppose in this paper that $A$ satisfies

$$
|A(x, t, \zeta)| \leq \Lambda_{1}|\zeta|
$$

and

$$
\langle A(x, t, \zeta)-A(x, t, \xi), \zeta-\xi\rangle \geq \Lambda_{2}|\zeta-\xi|^{2},
$$

for every $(\xi, \zeta) \in \mathbb{R}^{N} \times \mathbb{R}^{N}$ and a.e. $(x, t) \in \mathbb{R}^{N} \times \mathbb{R}$, where $\Lambda_{1}$ and $\Lambda_{2}$ are positive constants. In addtion, we also assume that the derivatives of $A$ with respect to $\zeta$ are bounded, that is,

$$
\left|A_{\zeta}(x, t, \zeta)\right| \leq \Lambda_{1},
$$

for any $\zeta \in \mathbb{R}^{N}$ and $(x, t) \in \mathbb{R}^{N}$. We remark that the condition (1.4) is needed in order to ensure that the reference problems (2.5) and (2.17) in the next section have $C^{0,1}$ regularity solutions (see $[11,12]$ ), which will be used in the sequel.

Throughout the paper, we assume that $A$ satisfies (1.2) and (1.3), (1.4). Besides, we always denote $T_{0}=\operatorname{diam}(\Omega)+T^{1 / 2}$ and $Q_{\rho}(x, t)=B_{\rho}(x) \times\left(t-\rho^{2}, t\right) \tilde{Q}_{\rho}(x, t)=$ $B_{\rho}(x) \times\left(t-\rho^{2} / 2, t+\rho^{2} / 2\right)$ for $(x, t) \in \mathbb{R}^{N+1}$ and $\rho>0$.

A weak solution $u$ of (1.1) is understood in the standard weak (distributional) sense, that is $u \in L^{1}\left(0, T, W_{0}^{1,1}(\Omega)\right)$ is a weak solution of (1.1) if

$$
-\int_{\Omega_{T}} u \varphi_{t} d x d t+\int_{\Omega_{T}} A(x, t, \nabla u) \nabla \varphi d x d t=-\int_{\Omega_{T}} F \nabla \varphi d x d t
$$

for all $\varphi \in C_{c}^{1}([0, T) \times \Omega)$.

The existence and uniqueness of weak solutions in $L^{2}\left(0, T, H_{0}^{1}(\Omega)\right)$ to problem $(1.1)$ with $F \in L^{2}\left(\Omega_{T}, \mathbb{R}^{N}\right)$ is given at the beginning of the next section.

For our purpose, we need a condition on $\Omega$ which is expressed in the following way. We say that $\Omega$ is a $\left(\delta, R_{0}\right)$-Reifenberg flat domain for $\delta \in(0,1)$ and $R_{0}>0$ if for every $x \in \partial \Omega$ and every $r \in\left(0, R_{0}\right]$, there exists a system of coordinates $\left\{z_{1}, z_{2}, \ldots, z_{n}\right\}$, which may depend on $r$ and $x$, so that in this coordinate system $x=0$ and that

$$
B_{r}(0) \cap\left\{z_{n}>\delta r\right\} \subset B_{r}(0) \cap \Omega \subset B_{r}(0) \cap\left\{z_{n}>-\delta r\right\} .
$$

We notice that this class of flat domains is rather wide since it includes $C^{1}$ domains, Lipschitz domains with sufficiently small Lipschitz constants and even fractal domains. Besides, it has many important roles in the theory of minimal surfaces and free boundary problems. This class appeared first in a work of Reifenberg (see [20]) in the context of Plateau problem. Its properties can be found in $[9,10,23]$.

We also require that the nonlinearity $A$ satisfies a smallness condition of BMO type in the $x$ variable in the sense that $A(x, t, \zeta)$ satisfies a $\left(\delta, R_{0}\right)$-BMO condition for some $\delta, R_{0}>0$ with exponent $p>0$ if

$$
[A]_{p}^{R_{0}}:=\sup _{(y, s) \in \mathbb{R}^{N} \times \mathbb{R}, 0<r \leq R_{0}}\left(f_{Q_{r}(y, s)}\left(\Theta\left(A, B_{r}(y)\right)(x, t)\right)^{p} d x d t\right)^{\frac{1}{p}} \leq \delta,
$$

where

$$
\Theta\left(A, B_{r}(y)\right)(x, t):=\sup _{\zeta \in \mathbb{R}^{N} \backslash\{0\}} \frac{\left|A(x, t, \zeta)-\bar{A}_{B_{r}(y)}(t, \zeta)\right|}{|\zeta|},
$$


and $\bar{A}_{B_{r}(y)}(t, \zeta)$ is denoted the average of $A(t, ., \zeta)$ over the ball $B_{r}(y)$, i.e,

$$
\bar{A}_{B_{r}(y)}(t, \zeta):=f_{B_{r}(y)} A(x, t, \zeta) d x=\frac{1}{\left|B_{r}(y)\right|} \int_{B_{r}(y)} A(x, t, \zeta) d x .
$$

The above condition appeared in our previous paper [19]. It is easy to see that the $\left(\delta, R_{0}\right)-\mathrm{BMO}$ is satisfied when $A$ is continuous or has small jump discontinuities with respect to $x$. We recall that a positive function $w \in L_{\text {loc }}^{1}\left(\mathbb{R}^{N+1}\right)$ is called an $\mathbf{A}_{p}$ weight, $1 \leq p<\infty$ if there holds

$$
\begin{aligned}
{[w]_{\mathbf{A}_{p}} } & :=\sup _{\tilde{Q}_{\rho}(x, t) \subset \mathbb{R}^{N+1}}\left(f_{\tilde{Q}_{\rho}(x, t)} w(y, s) d y d s\right)\left(f_{\tilde{Q}_{\rho}(x, t)} w(y, s)^{-\frac{1}{p-1}} d y d s\right)^{p-1} \\
& <\infty \text { when } p>1
\end{aligned}
$$

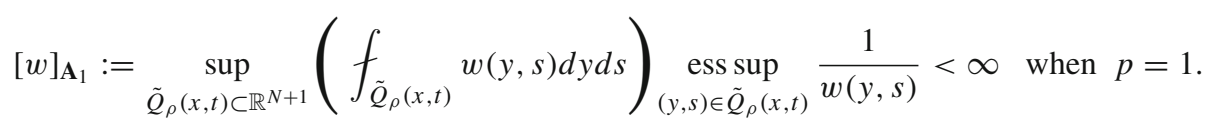

The $[w]_{\mathbf{A}_{p}}$ is called the $\mathbf{A}_{p}$ constant of $w$.

A positive function $w \in L_{\text {loc }}^{1}\left(\mathbb{R}^{N+1}\right)$ is called an $\mathbf{A}_{\infty}$ weight if there are two positive constants $C$ and $v$ such that

$$
w(E) \leq C\left(\frac{|E|}{|Q|}\right)^{v} w(Q)
$$

for all cylinder $Q=\tilde{Q}_{\rho}(x, t)$ and all measurable subsets $E$ of $Q$. The pair $(C, v)$ is called the $\mathbf{A}_{\infty}$ constant of $w$ and is denoted by $[w]_{\mathbf{A}_{\infty}}$.

It is well known that this class is the union of $\mathbf{A}_{p}$ for all $p \in(1, \infty)$, see [7]. If $w$ is a weight function belonging to $w \in \mathbf{A}_{\infty}$ and $E \subset \mathbb{R}^{N+1}$ a Borel set, $0<q<\infty, 0<s \leq \infty$, the weighted Lorentz space $L_{w}^{q, s}(E)$ is the set of measurable functions $g$ on $E$ such that

$$
\|g\|_{L_{w}^{q, s}(E)}:= \begin{cases}\left(q \int_{0}^{\infty}\left(\rho^{q} w(\{(x, t) \in E:|g(x, t)|>\rho\})\right)^{\frac{s}{q}} \frac{d \rho}{\rho}\right)^{1 / s}<\infty & \text { if } s<\infty, \\ \sup _{\rho>0} \rho(w(\{(x, t) \in E:|g(x, t)|>\rho\}))^{1 / q}<\infty & \text { if } s=\infty .\end{cases}
$$

Here we write $w(O)=\int_{O} w(x, t) d x d t$ for a measurable set $O \subset \mathbb{R}^{N+1}$. Obviously, $\|g\|_{L_{w}^{q, q}(E)}=\|g\|_{L_{w}^{q}(E)}$, thus $L_{w}^{q, q}(E)=L_{w}^{q}(E)$. As usual, when $w \equiv 1$ we write simply $L^{q, s}(E)$ instead of $L_{w}^{q, s}(E)$. In this paper, $\mathcal{M}$ denotes the parabolic Hardy-Littlewood maximal function defined for each locally integrable function $f$ in $\mathbb{R}^{N+1}$ by

$$
\mathcal{M}(f)(x, t)=\sup _{\rho>0} f_{\tilde{Q}_{\rho}(x, t)}|f(y, s)| d y d s \quad \forall(x, t) \in \mathbb{R}^{N+1} .
$$

If $p>1$ and $w \in \mathbf{A}_{p}$ we verify that $\mathcal{M}$ is operator from $L^{1}\left(\mathbb{R}^{N+1}\right)$ into $L^{1, \infty}\left(\mathbb{R}^{N+1}\right)$ and $L_{w}^{p, s}\left(\mathbb{R}^{N+1}\right)$ into itself for $0<s \leq \infty$, see [21,22,24].

We would like to mention that the use of the Hardy-Littlewood maximal function in nonlinear degenerate problems was started in the elliptic setting by T. Iwaniec in his fundamental paper [8]. We now state the main result of the paper.

Theorem 1.1 Let $F \in L^{2}\left(\Omega_{T}, \mathbb{R}^{N}\right)$. There exists a unique weak solution $u \in$ $L^{2}\left(0, T, H_{0}^{1}(\Omega)\right)$ of (1.1). For any $w \in \mathbf{A}_{\infty}, 0<q<\infty, 0<s \leq \infty$ we find 
$\delta=\delta\left(N, \Lambda_{1}, \Lambda_{2}, q, s,[w]_{\mathbf{A}_{\infty}}\right) \in(0,1)$ and $s_{0}=s_{0}\left(N, \Lambda_{1}, \Lambda_{2}\right)>0$ such that if $\Omega$ is $\left(\delta, R_{0}\right)$-Reifenberg flat domain $\Omega$ and $[A]_{s_{0}}^{R_{0}} \leq \delta$ for some $R_{0}>0$ then

$$
\left\|\mathcal{M}\left(|\nabla u|^{2}\right)\right\|_{L_{w}^{q, s}\left(\Omega_{T}\right)} \leq C|| \mathcal{M}\left(|F|^{2}\right) \|_{L_{w}^{q, s}\left(\Omega_{T}\right)} .
$$

Here C depends only on $N, \Lambda_{1}, \Lambda_{2}, q, s,[w]_{\mathbf{A}_{\infty}}$ and $T_{0} / R_{0}$.

Since $\mathcal{M}$ is a bounded operator from $L_{w}^{p, s}\left(\mathbb{R}^{N+1}\right)$ into itself for $p>1,0<s \leq \infty$ and $w \in \mathbf{A}_{p}$, thus we obtain the following Theorem.

Theorem 1.2 Let $F \in L^{2}\left(\Omega_{T}, \mathbb{R}^{N}\right)$ and $s_{0}$ be in Theorem 1.1. There exists a unique weak solution $u \in L^{2}\left(0, T, H_{0}^{1}(\Omega)\right)$ of (1.1). For any $w \in \mathbf{A}_{q / 2}, 2<q<\infty, 0<s \leq \infty$ we find $\delta=\delta\left(N, \Lambda_{1}, \Lambda_{2}, q, s,[w]_{\mathbf{A}_{q / 2}}\right) \in(0,1)$ and such that if $\Omega$ is $\left(\delta, R_{0}\right)$-Reifenberg flat domain and $[A]_{s_{0}}^{R_{0}} \leq \delta$ for some $R_{0}>0$ then

$$
\||| \nabla u\|_{L_{w}^{q, s}\left(\Omega_{T}\right)} \leq\left. C|\| F|\right|_{L_{w}^{q, s}\left(\Omega_{T}\right)} .
$$

Here $C$ depends only on $N, \Lambda_{1}, \Lambda_{2}, q, s,[w]_{\mathbf{A}_{q / 2}}$ and $T_{0} / R_{0}$.

We remark that the global gradient estimates of solutions of (1.1) obtained in Theorem 1.2 extend results in [2-4] to more general nonlinear structure and in the setting of weighted Lorentz spaces. Notice that Theorems 1.1 and 1.2 in the quasilinear elliptic framework are obtained in [14]. In the linear case, we obtain global estimates for gradients of weak solutions to problem

$$
\left\{\begin{array}{l}
u_{t}-\operatorname{div}(A(x, t, \nabla u))=\operatorname{div}(F)+\mu \text { in } \Omega_{T}, \\
u=0 \text { on } \partial \Omega \times(0, T), \\
u(0)=\sigma \text { in } \Omega
\end{array}\right.
$$

where $F \in L^{1}\left(\Omega_{T}, \mathbb{R}^{N}\right), \mu \in \mathfrak{M}_{b}\left(\Omega_{T}\right)$ the set of bounded Radon measure in $\Omega_{T}, \sigma \in$ $\mathfrak{M}_{b}(\Omega)$ the set of bounded Radon measure in $\Omega$.

Theorem 1.3 Suppose that $A$ is linear. Let $F \in L^{1}\left(\Omega_{T}, \mathbb{R}^{N}\right), \mu \in \mathfrak{M}_{b}\left(\Omega_{T}\right), \sigma \in \mathfrak{M}_{b}(\Omega)$, set $\omega=|\mu|+|\sigma| \otimes \delta_{\{t=0\}}$. Let $s_{0}$ be as in Theorem 1.1.

(a) For any $q>2,0<s \leq \infty, w \in \mathbf{A}_{q / 2}$ and $\mathcal{M}_{1}[\omega],|F| \in L_{w}^{q, s}\left(\Omega_{T}\right)$ we find a $\delta=$ $\delta\left(N, \Lambda_{1}, \Lambda_{2}, q, s,[w]_{\mathbf{A}_{q / 2}}\right) \in(0,1)$ such that if $\Omega$ is a $\left(\delta, R_{0}\right)$-Reifenberg flat domain and $[A]_{s_{0}}^{R_{0}} \leq \delta$ for some $R_{0}>0$ there exists a unique weak solution $u \in L^{2}\left(0, T, H_{0}^{1}(\Omega)\right)$ of (1.8) and there holds

$$
\left\|\left|\nabla u\|\|_{L_{w}^{q, s}\left(\Omega_{T}\right)} \leq C\left\|\mathcal{M}_{1}[\omega]\right\|_{L_{w}^{q, s}\left(\Omega_{T}\right)}+C\right|\right\| F \|\left.\right|_{L_{w}^{q, s}\left(\Omega_{T}\right)},
$$

where $C$ depends only on $N, \Lambda_{1}, \Lambda_{2}, q, s,[w]_{\mathbf{A}_{q / 2}}$ and $T_{0} / R_{0}$.

(b) For any $\varepsilon \in(0,1), \frac{2(\varepsilon+1)}{\varepsilon+2}<q \leq 2,0<s \leq \infty, w^{2+\varepsilon} \in \mathbf{A}_{1}$ and $\mathcal{M}_{1}[\omega],|F| \in$ $L_{w}^{q, s}\left(\Omega_{T}\right)$ we find $a \delta=\delta\left(N, \Lambda_{1}, \Lambda_{2}, q, s, \varepsilon,\left[w^{2+\varepsilon}\right]_{\mathbf{A}_{1}}\right) \in(0,1)$ such that if $\Omega$ is a $\left(\delta, R_{0}\right)$-flat domain and $[A]_{s_{0}}^{R_{0}} \leq \delta$ for some $R_{0}>0$ there exists a unique weak solution $u \in L^{\frac{2(\varepsilon+1)}{\varepsilon+2}}\left(0, T, W_{0}^{1, \frac{2(\varepsilon+1)}{\varepsilon+2}}(\Omega)\right)$ of (1.8) and there holds

$$
\||| \nabla u\|\left\|_{L_{w}^{q, s}\left(\Omega_{T}\right)} \leq C\right\| \mathcal{M}_{1}[\omega]\left\|_{L_{w}^{q, s}\left(\Omega_{T}\right)}+C|\|F\||_{L_{w}^{q, s}\left(\Omega_{T}\right)},\right.
$$

where $C$ depends only on $N, \Lambda_{1}, \Lambda_{2}, q, s, \varepsilon,\left[w^{2+\varepsilon}\right]_{\mathbf{A}_{1}}$ and $T_{0} / R_{0}$. 
In above Theorem, $\mathcal{M}_{1}$ denotes the first order fractional Maximal parabolic potential on $\mathbb{R}^{N+1}$ of a positive Radon measure in $\mathbb{R}^{N+1}$ by

$$
\mathcal{M}_{1}[\mu](x, t)=\sup _{0<\rho<R} \frac{\mu\left(\tilde{Q}_{\rho}(x, t)\right)}{\rho^{N+1}} \forall(x, t) \in \mathbb{R}^{N+1} .
$$

We can use estimates (1.7) in Theorem 1.2 and (1.9)-(1.10) in Theorem 1.3 and the following Lemma to get upper bounds for gradients of the solutions in Lorentz-Morrey spaces.

Lemma 1.4 Let $0<q<\infty, 0<s \leq \infty, \gamma \geq 1$ and $H_{1}, H_{2}$ be measurable functions in $\Omega_{T}$. If

$$
\left\|H_{1}\right\|_{L_{w}^{q, s}\left(\Omega_{T}\right)} \leq C\left(N, q, s,\left[w^{\gamma}\right]_{\mathbf{A}_{1}}\right)\left\|H_{2}\right\|_{L_{w}^{q, s}\left(\Omega_{T}\right)},
$$

for any $w^{\gamma} \in \mathbf{A}_{1}$, then for any $\kappa \in\left(\frac{(N+2)(\gamma-1)}{\gamma}, N+2\right], \vartheta \in\left(\frac{N(\gamma-1)}{\gamma}, N\right]$,

$$
\left\|H_{1}\right\|_{L_{*}^{q, s ; \kappa}\left(\Omega_{T}\right)} \leq C(N, q, s, \gamma, \kappa)\left\|H_{2}\right\|_{L_{*}^{q, s ; \kappa}\left(\Omega_{T}\right)},
$$

and

$$
\left\|H_{1}\right\|_{L_{* *}^{q, s ; \vartheta}\left(\Omega_{T}\right)} \leq C(N, q, s, \gamma, \vartheta)\left\|H_{2}\right\|_{L_{* *}^{q, s ; \vartheta}\left(\Omega_{T}\right)} .
$$

In (1.11), $L_{*}^{q, s ; \kappa}\left(\Omega_{T}\right)$ denotes Lorentz-Morrey space, is the set of measurable functions $g$ in $\Omega_{T}$ such that

$$
\|g\|_{L_{*}^{q, s ; \kappa}\left(\Omega_{T}\right)}:=\sup _{0<\rho<T_{0},(x, t) \in \Omega_{T}} \rho^{\frac{\kappa-N-2}{q}}\|g\|_{L^{q, s}\left(\tilde{Q}_{\rho}(x, t) \cap \Omega_{T}\right)}<\infty .
$$

In (1.12), $L_{* *}^{q, s ; \vartheta}\left(\Omega_{T}\right)$ is the Lorentz-Morrey space of measurable functions $g$ in $\Omega_{T}$ such that

$$
\|g\|_{L_{* *}^{q, s ; \vartheta}\left(\Omega_{T}\right)}:=\sup _{0<\rho<\operatorname{diam}(\Omega), x \in \Omega} \rho^{\frac{\vartheta-N}{q}}\|g\|_{L^{q, s}\left(\left(B_{\rho}(x) \cap \Omega\right) \times(0, T)\right)}<\infty .
$$

This Lemma is inspired by [13, Proof of Theorem 2.3], its proof can be found in [19, Proof of Theorem 2.21] and notice that for $\left(x_{0}, t_{0}\right) \in \Omega_{T}$ and $0<\rho<T_{0}$

$$
\begin{aligned}
& w_{1}(x, t)=\min \left\{\rho^{-N-2+\kappa-\kappa_{1}}, \max \left\{\left|x-x_{0}\right|, \sqrt{2\left|t-t_{0}\right|}\right\}^{-N-2+\kappa-\kappa_{1}}\right\}, \\
& w_{2}(x, t)=\min \left\{\rho^{-N+\vartheta-\vartheta_{1}},\left|x-x_{0}\right|^{-N+\vartheta-\vartheta_{1}}\right\},
\end{aligned}
$$

where $0<\kappa_{1}<\kappa-\frac{(N+2)(\gamma-1)}{\gamma}, 0<\vartheta_{1}<\kappa-\frac{N(\gamma-1)}{\gamma}$ and

$$
\left[w_{1}^{\gamma}\right]_{\mathbf{A}_{1}} \leq C\left(N, \kappa_{1}, \kappa, \gamma\right), \quad\left[w_{2}^{\gamma}\right]_{\mathbf{A}_{1}} \leq C\left(N, \vartheta_{1}, \vartheta, \gamma\right) .
$$

For example, from (1.9) in Theorem 1.3 and Lemma 1.4 we obtain for $2<q<\infty$, $0<s \leq \infty$ and $0<\kappa \leq N+2,0<\vartheta \leq N+2$ there hold

$$
\begin{aligned}
& \left\|\left.\left|\nabla u\left\|\left.\right|_{L_{*}^{q, s ; \kappa}\left(\Omega_{T}\right)} \leq C\right\| \mathcal{M}_{1}[|\omega|]\left\|_{L_{*}^{q, s ; \kappa}\left(\Omega_{T}\right)}+C\right\|\right||F|\right|_{L_{*}^{q, s ; \kappa}\left(\Omega_{T}\right)},\right. \\
& \left\|\left|\nabla u\left\|\left.\right|_{L_{* *}^{q, s ; \vartheta}\left(\Omega_{T}\right)} \leq C\right\| \mathcal{M}_{1}[\omega]\left\|_{L_{* *}^{q, s ; \vartheta}\left(\Omega_{T}\right)}+C|||F|\right\|_{L_{* *}^{q, s ; \vartheta}\left(\Omega_{T}\right)},\right.\right.
\end{aligned}
$$

and from (1.10) in Theorem 1.3 we also have preceding estimates with $1<q \leq 2,0<s \leq \infty$ and $\frac{N+2}{2}<\kappa \leq N+2, \frac{N}{2}<\vartheta \leq N$. Furthermore, according to [19, Proof of Theorem 2.21] we verify that for $q>1,0<\vartheta<\min \{N, q\}$ and $\varphi \in L^{1}\left(0, T, W_{0}^{1,1}(\Omega)\right)$ there holds

$$
\left(\int_{0}^{T}\left|\operatorname{osc}_{B_{\rho} \cap \bar{\Omega}} \varphi(t)\right|^{q} d t\right)^{\frac{1}{q}} \leq C \rho^{1-\frac{\vartheta}{q}}|| \mid \nabla \varphi \|_{L_{* *}^{q, q ; \vartheta}\left(\Omega_{T}\right)},
$$


for any ball $B_{\rho} \subset \mathbb{R}^{N}$, where $C=C(N, q, \vartheta)$. Therefore, (1.13) implies a global Holderestimate in space variable and $L^{q}$-estimate in time, namely for all ball $B_{\rho} \subset \mathbb{R}^{N}$

$$
\left(\int_{0}^{T}\left|\operatorname{osc}_{B_{\rho} \cap \bar{\Omega}} u(t)\right|^{q} d t\right)^{\frac{1}{q}} \leq C \rho^{1-\frac{\vartheta}{q}}\left(|| \mathcal{M}_{1}[\omega]||_{L_{* *}^{q, q ; \vartheta}\left(\Omega_{T}\right)}+\left.|||F|\right|_{L_{* *}^{q, q ; \vartheta}\left(\Omega_{T}\right)}\right),
$$

with $0<\vartheta<\min \{N, q\}$.

We would like to refer to $[16,17]$ as the first papers which have been used the first order factional maximal operators in order to obtain the Lorentz-Morrey estimates for gradients of solutions to nonlinear elliptic equations with measure or $L^{1}$ data.

Finally, we use Theorem 1.3 to prove the existence of solutions of the Riccati type parabolic equations

$$
\left\{\begin{array}{l}
u_{t}-\operatorname{div}(A(x, t, \nabla u))=|\nabla u|^{q}+\operatorname{div}(F)+\mu \text { in } \Omega_{T}, \\
u=0 \text { on } \partial \Omega \times(0, T), \\
u(0)=\sigma \quad \text { in } \Omega
\end{array}\right.
$$

where $q>1$ and $F \in L^{q}\left(\Omega_{T}, \mathbb{R}^{N}\right), \mu \in \mathfrak{M}_{b}\left(\Omega_{T}\right), \sigma \in \mathfrak{M}_{b}(\Omega)$.

Theorem 1.5 Suppose that $A$ is linear. Let $q>1, F \in L^{q}\left(\Omega_{T}, \mathbb{R}^{N}\right)$ and $\mu \in \mathfrak{M}_{b}\left(\Omega_{T}\right), \sigma \in$ $\mathfrak{M}_{b}(\Omega)$, set $\omega=|\mu|+|\sigma| \otimes \delta_{\{t=0\}}$. There exist $C_{1}=C_{1}\left(N, \Lambda_{1}, \Lambda_{2}, q, T_{0}\right), \delta=$ $\delta\left(N, \Lambda_{1}, \Lambda_{2}, q\right) \in(0,1)$ and $s_{0}=s_{0}\left(N, \Lambda_{1}, \Lambda_{2}\right)>0$ such that if $\Omega$ is a $\left(\delta, R_{0}\right)$-Reifenberg flat domain and $[A]_{s_{0}}^{R_{0}} \leq \delta$ for some $R_{0}>0$ and

$$
\omega(K) \leq C_{1} \operatorname{Cap}_{\mathcal{G}_{1}, q^{\prime}}(K),
$$

and

$$
\int_{K} H_{q} d x d t \leq C_{1}^{q} \operatorname{Cap}_{\mathcal{G}_{1}, q^{\prime}}(K)
$$

for any compact set $K \subset \mathbb{R}^{N}$ where $H_{q}=\left(\mathcal{M}\left(|F|^{2}\right)\right)^{q / 2} \chi_{\Omega_{T}}$ if $q \geq \frac{N+2}{N}$ and $H_{q}=$ $|F|^{q} \chi_{\Omega_{T}}$ if $q<\frac{N+2}{N}$, then problem (1.15) has a weak solution $u \in L^{q}\left(0, T, W_{0}^{1, q}(\Omega)\right)$ satisfying

$$
\int_{K \cap \Omega_{T}}|\nabla u|^{q} d x d t \leq C_{2} \operatorname{Cap}_{\mathcal{G}_{1}, q^{\prime}}(K),
$$

for any compact set $K \subset \mathbb{R}^{N}$, here $C_{2}=C_{2}\left(N, \Lambda_{1}, \Lambda_{2}, q, T_{0} / R_{0}, T_{0}, C_{1}\right)>0$.

In this Theorem, capacity $\operatorname{Cap}_{\mathcal{G}_{1}, q^{\prime}}$ denotes the $\left(\mathcal{G}_{1}, q^{\prime}\right)$-capacity where $\mathcal{G}_{1}$ is the Bessel parabolic kernel of first order (see [1])

$$
\mathcal{G}_{1}(x, t)=\left((4 \pi)^{N / 2} \Gamma(1 / 2)\right)^{-1} \frac{\chi_{(0, \infty)}(t)}{t^{(N+1) / 2}} \exp \left(-t-\frac{|x|^{2}}{4 t}\right) \text { for }(x, t) \text { in } \mathbb{R}^{N+1} .
$$

It is defined by

$$
\operatorname{Cap}_{\mathcal{G}_{1}, q^{\prime}}(E)=\inf \left\{\int_{\mathbb{R}^{N+1}}|f|^{q^{\prime}} d x d t: f \in L_{+}^{q^{\prime}}\left(\mathbb{R}^{N+1}\right), \mathcal{G}_{1} * f \geq \chi_{E}\right\},
$$

for any Borel set $E \subset \mathbb{R}^{N+1}$, where $\chi_{E}$ is the characteristic function on $E$.

Note that if $1<q<\frac{N+2}{N+1}$, the capacity $\operatorname{Cap}_{\mathcal{G}_{1}, q^{\prime}}$ of a singleton is positive thus (1.16) and (1.17) hold for some constant $C_{1}>0$ provided $\mu \in \mathfrak{M}_{b}\left(\Omega_{T}\right), u_{0} \in \mathfrak{M}_{b}(\Omega)$ and $|F| \in L^{q}\left(\Omega_{T}\right)$. We remark that in case $F \equiv 0$ the existence of solutions to (1.15) has been obtained in our paper [19]. 
Remark 1.6 The inequality (1.16) is equivalent to

$$
\begin{aligned}
& |\mu|(K) \leq C \operatorname{Cap}_{\mathcal{G}_{1}, q^{\prime}}(K), \quad \sigma \equiv 0 \quad \text { when } q \geq 2, \\
& |\mu|(K) \leq C \operatorname{Cap}_{\mathcal{G}_{1}, q^{\prime}}(K), \quad|\sigma|(O) \leq \operatorname{Cap}_{\frac{\mathbf{G}_{\frac{2-q}{q}}, q^{\prime}}{q}}(O) \quad \text { when } 1<q<2,
\end{aligned}
$$

for any compact sets $K \subset \mathbb{R}^{N+1}, O \subset \mathbb{R}^{N}$, where $\mathbf{G}_{\frac{2-q}{q}}$ is the Bessel kernel of order $\frac{2-q}{q}$ and capacity $\operatorname{Cap}_{\mathbf{G}_{\frac{2-q}{q}}, q^{\prime}}$ of $O$ is defined by

$$
\operatorname{Cap}_{\mathbf{G}_{\frac{2-q}{q}}, q^{\prime}}(O)=\inf \left\{\int_{\mathbb{R}^{N}}|f|^{q^{\prime}} d x: f \in L_{+}^{q^{\prime}}\left(\mathbb{R}^{N}\right), \mathbf{G}_{\frac{2-q}{q}} * f \geq \chi_{O}\right\},
$$

see [19, Remark 4.34]. Moreover, if $q>2$, the inequality (1.17) is equivalent to

$$
\int_{K \cap \Omega_{T}}|F|^{q} d x d t \leq C \operatorname{Cap}_{\mathcal{G}_{1}, q^{\prime}}(K)
$$

for any compact set $K \subset \mathbb{R}^{N}$, see Lemma 4.1.

\section{Interior estimates and boundary estimates for parabolic equations}

In this section we present various local interior and boundary estimates for weak solution $u$ of (1.1). They will be used for our global estimates later. First we recall basic existence and uniqueness result of problem (1.1).

Proposition 1 If $F \in L^{2}\left(\Omega_{T}, \mathbb{R}^{N}\right)$, there exists a unique weak solution $u \in$ $L^{2}\left(0, T ; H_{0}^{1}(\Omega)\right)$ of $(1.1)$ and the following global estimate holds:

$$
\int_{\Omega_{T}}|\nabla u|^{2} d x d t \leq \Lambda_{2}^{-1 / 2} \int_{\Omega_{T}}|F|^{2} d x d t .
$$

The existence and uniqueness of a weak solution of problem (1.1) with $F \in L^{2}\left(\Omega_{T}, \mathbb{R}^{N}\right)$ is obtained from the Lax-Milgram Theorem, version for parabolic framework. Using $u$ as a test function in (1.1), we get (2.1). Moreover, due to the embedding

$$
\left\{\varphi: \varphi \in L^{2}\left(0, T ; H_{0}^{1}(\Omega)\right), \varphi_{t} \in L^{2}\left(0, T ; H^{-1}(\Omega)\right)\right\} \subset C\left(0, T ; L^{2}(\Omega)\right),
$$

thus, the unique weak solution $u$ of (1.1) belongs to $C\left(0, T ; L^{2}(\Omega)\right)$. We can see that $u$ is also the unique weak solution of (1.1) in $\Omega \times(-\infty, T)$ where $F \in L^{2}\left(\Omega_{T}, \mathbb{R}^{N}\right)$ and $F=0, u=0$ in $\Omega \times(-\infty, 0)$.

For some technical reasons, throughout this section, we always assume that $u \in$ $C\left(-\infty, T ; L^{2}(\Omega)\right) \cap L^{2}\left(-\infty, T ; H_{0}^{1}(\Omega)\right)$ is a weak solution to equation (1.1) in $\Omega \times$ $(-\infty, T)$ with $F \in L^{2}\left(\Omega_{T}, \mathbb{R}^{N}\right), F=0$ in $\Omega \times(-\infty, 0)$.

\subsection{Interior estimates}

For each ball $B_{2 R}=B_{2 R}\left(x_{0}\right) \subset \subset \Omega$ and $t_{0} \in(0, T)$, one considers the unique solution

$$
w \in C\left(t_{0}-4 R^{2}, t_{0} ; L^{2}\left(B_{2 R}\right)\right) \cap L^{2}\left(t_{0}-4 R^{2}, t_{0} ; H^{1}\left(B_{2 R}\right)\right)
$$

to the following equation

$$
\left\{\begin{array}{l}
w_{t}-\operatorname{div}(A(x, t, \nabla w))=0 \quad \text { in } Q_{2 R}, \\
w=u \quad \text { on } \partial_{p} Q_{2 R}
\end{array}\right.
$$


where $Q_{2 R}=B_{2 R} \times\left(t_{0}-4 R^{2}, t_{0}\right)$ and $\partial_{p} Q_{2 R}=\left(\partial B_{2 R} \times\left(t_{0}-4 R^{2}, t_{0}\right)\right) \cup\left(B_{2 R} \times\right.$ $\left.\left\{t=t_{0}-4 R^{2}\right\}\right)$.

The following a variant of Gehring's lemma was proved in $[6,18]$.

Lemma 2.1 Let $w$ be in (2.2). There exist constants $\theta_{1}>2$ and $C$ depending only on $N, \Lambda_{1}, \Lambda_{2}$ such that the following estimate

$$
\left(f_{Q_{\rho / 2}(y, s)}|\nabla w|^{\theta_{1}} d x d t\right)^{\frac{1}{\theta_{1}}} \leq C f_{Q_{\rho}(y, s)}|\nabla w| d x d t
$$

holds for all $Q_{\rho}(y, s) \subset Q_{2 R}$.

The next lemma gives an estimate for $\nabla u-\nabla w$.

Lemma 2.2 Let $w$ be in (2.2). There exists a constant $C=C\left(N, \Lambda_{1}, \Lambda_{2}\right)>0$ such that

$$
f_{Q_{2 R}}|\nabla u-\nabla w|^{2} d x d t \leq C f_{Q_{2 R}}|F|^{2} d x d t .
$$

Proof Using $u-w$ as a test function in (1.1) and (2.2) and since

$$
\int_{Q_{2 R}} u_{t}(u-w) d x d t-\int_{Q_{2 R}} w_{t}(u-w) d x d t=\frac{1}{2} \int_{B_{2 R}}(u-w)^{2}\left(t_{0}\right) d x \geq 0,
$$

we find

$$
\int_{Q_{2 R}}\langle A(x, t, \nabla u)-A(x, t, \nabla w), \nabla u-\nabla w\rangle d x d t \leq \int_{Q_{2 R}}\langle F, \nabla u-\nabla w\rangle d x d t .
$$

Using (1.3) and Hölder inequality we derive (2.4).

To continue, we denote by $v$ the unique function

$$
v \in C\left(t_{0}-R^{2}, t_{0} ; L^{2}\left(B_{R}\right)\right) \cap L^{2}\left(t_{0}-R^{2}, t_{0} ; H^{1}\left(B_{R}\right)\right)
$$

solution of the following equation

$$
\left\{\begin{array}{l}
v_{t}-\operatorname{div}\left(\bar{A}_{B_{R}\left(x_{0}\right)}(t, \nabla v)\right)=0 \text { in } Q_{R}, \\
v=w \text { on } \partial_{p} Q_{R}
\end{array}\right.
$$

where $Q_{R}=B_{R}\left(x_{0}\right) \times\left(t_{0}-R^{2}, t_{0}\right)$ and $\partial_{p} Q_{R}=\left(\partial B_{R} \times\left(t_{0}-R^{2}, t_{0}\right)\right) \cup\left(B_{R} \times\right.$ $\left.\left\{t=t_{0}-R^{2}\right\}\right)$.

Lemma 2.3 Let $\theta_{1}$ be the constant in Lemma 2.1. There exist constants $C_{1}=C_{1}\left(N, \Lambda_{1}, \Lambda_{2}\right)$ and $C_{2}=C_{2}\left(\Lambda_{1}, \Lambda_{2}\right)$ such that

$$
f_{Q_{R}}|\nabla w-\nabla v|^{2} d x d t \leq C_{1}\left([A]_{S_{1}}^{R}\right)^{2} f_{Q_{2 R}}|\nabla w|^{2} d x d t,
$$

with $s_{1}=\frac{2 \theta_{1}}{\theta_{1}-2}$ and

$$
C_{2}^{-1} \int_{Q_{R}}|\nabla v|^{2} d x d t \leq \int_{Q_{R}}|\nabla w|^{2} d x d t \leq C_{2} \int_{Q_{R}}|\nabla v|^{2} d x d t .
$$

Proof The proof can be found in [19, Lemma 7.3]. 
Theorem 2.4 Let $\theta_{1}$ be the constant in Lemma 2.1. There exists a functions $v \in C\left(t_{0}-\right.$ $\left.R^{2}, t_{0} ; L^{2}\left(B_{R}\right)\right) \cap L^{2}\left(t_{0}-R^{2}, t_{0} ; H^{1}\left(B_{R}\right)\right) \cap L^{\infty}\left(t_{0}-\frac{1}{4} R^{2}, t_{0} ; W^{1, \infty}\left(B_{R / 2}\right)\right)$ such that

$$
\|\nabla v\|_{L^{\infty}\left(Q_{R / 2}\right)}^{2} \leq C f_{Q_{2 R}}|\nabla u|^{2} d x d t+C f_{Q_{2 R}}|F|^{2} d x d t
$$

and

$$
\begin{aligned}
& f_{Q_{R}}|\nabla u-\nabla v|^{2} d x d t \leq C f_{Q_{2 R}}|F|^{2} d x d t+C\left([A]_{S_{1}}^{R}\right)^{2} \\
& \times \quad\left(f_{Q_{2 R}}|\nabla u|^{2} d x d t+f_{Q_{2 R}}|F|^{2} d x d t\right),
\end{aligned}
$$

where $s_{1}=\frac{2 \theta_{1}}{\theta_{1}-2}$ and $C=C\left(N, \Lambda_{1}, \Lambda_{2}\right)$.

Proof Let $w$ and $v$ be in Eqs. (2.2) and (2.5). By standard interior regularity and inequality (2.3) in Lemma 2.1 and (2.7) in Lemma 2.3 we have

$$
\begin{aligned}
\|\nabla v\|_{L^{\infty}\left(Q_{R / 2}\right)} & \leq C\left(f_{Q_{R}}|\nabla v|^{2} d x d t\right)^{1 / 2} \\
& \leq C\left(f_{Q_{R}}|\nabla w|^{2} d x d t\right)^{1 / 2} .
\end{aligned}
$$

Thus, we get (2.8) from inequality (2.4) in Lemma 2.2 .

On the other hand, applying (2.6) in Lemma 2.3 yields

$$
f_{Q_{R}}|\nabla u-\nabla v|^{2} d x d t \leq f_{Q_{R}}|\nabla u-\nabla w|^{2} d x d t+c_{4}\left([A]_{S_{1}}^{R}\right)^{2} f_{Q_{2 R}}|\nabla w|^{2} d x d t .
$$

Hence, we get (2.9) from (2.4) in Lemma 2.2. The proof is complete.

\subsection{Boundary estimates}

In this subsection, we focus on the corresponding estimates near the boundary.

Throughout this subsection, we always assume that $\Omega$ is a $\left(\delta, R_{0}\right)$ - Reifenberg flat domain with $0<\delta \leq 1 / 2$. In particular, we can see that the complement of $\Omega$ is uniformly 2 -thick for some constants $c_{0}, r_{0}$, see [19]. Let $x_{0} \in \partial \Omega$ be a boundary point and $0<R<R_{0} / 6$ and $t_{0} \in(0, T)$, we set $\tilde{\Omega}_{6 R}=\tilde{\Omega}_{6 R}\left(x_{0}, t_{0}\right)=\left(\Omega \cap B_{6 R}\left(x_{0}\right)\right) \times\left(t_{0}-(6 R)^{2}, t_{0}\right)$ and $Q_{6 R}=Q_{6 R}\left(x_{0}, t_{0}\right)$.

We now consider the unique solution $w$ to the equation

$$
\left\{\begin{array}{l}
w_{t}-\operatorname{div}(A(x, t, \nabla w))=0 \quad \text { in } \tilde{\Omega}_{6 R}, \\
w=u \text { on } \partial_{p} \tilde{\Omega}_{6 R} .
\end{array}\right.
$$

In what follows we extend $F$ and $u$ by zero to $(\Omega \times(-\infty, T))^{c}$ and then extend $w$ by $u$ to $\mathbb{R}^{N+1} \backslash \tilde{\Omega}_{6 R}$.

Lemma 2.5 Let $w$ be in (2.10). There exist constants $\theta_{2}>2$ and $C>0$ depending only on $N, \Lambda_{1}, \Lambda_{2}$ such that the following estimate

$$
\left(f_{Q_{\rho / 2}(y, s)}|\nabla w|^{\theta_{2}} d x d t\right)^{\frac{1}{\theta_{2}}} \leq C f_{Q_{3 \rho}(y, s)}|\nabla w| d x d t
$$

holds for all $Q_{3 \rho}(z, s) \subset Q_{6 R}$. 
Above lemma was proved in [19, Theorem 7.5]. Analogous to Lemma 2.2 we obtain

Lemma 2.6 Let $w$ be in (2.10). There exists a constant $C=C\left(N, \Lambda_{1}, \Lambda_{2}\right)>0$ such that

$$
f_{Q_{6 R}}|\nabla u-\nabla w|^{2} d x d t \leq C f_{Q_{6 R}}|F|^{2} d x d t .
$$

Next, we set $\rho=R(1-\delta)$ so that $0<\rho /(1-\delta)<R_{0} / 6$. By the definition of Reifenberg flat domains, there exists a coordinate system $\left\{y_{1}, y_{2}, \ldots, y_{N}\right\}$ with the origin $0 \in \Omega$ such that in this coordinate system $x_{0}=(0, \ldots, 0,-\rho \delta /(1-\delta))$ and

$$
B_{\rho}^{+}(0) \subset \Omega \cap B_{\rho}(0) \subset B_{\rho}(0) \cap\left\{y=\left(y_{1}, y_{2}, \ldots, y_{N}\right): y_{N}>-2 \rho \delta /(1-\delta)\right\} .
$$

Since $\delta<1 / 2$ we have

$$
B_{\rho}^{+}(0) \subset \Omega \cap B_{\rho}(0) \subset B_{\rho}(0) \cap\left\{y=\left(y_{1}, y_{2}, \ldots, y_{N}\right): y_{N}>-4 \rho \delta\right\},
$$

where $B_{\rho}^{+}(0):=B_{\rho}(0) \cap\left\{y=\left(y_{1}, y_{2}, \ldots, y_{N}\right): y_{N}>0\right\}$. Furthermore we consider the unique solution

$$
v \in C\left(t_{0}-\rho^{2}, t_{0} ; L^{2}\left(\Omega \cap B_{\rho}(0)\right)\right) \cap L^{2}\left(t_{0}-\rho^{2}, t_{0} ; H^{1}\left(\Omega \cap B_{\rho}(0)\right)\right)
$$

to the following equation

$$
\left\{\begin{array}{l}
v_{t}-\operatorname{div}\left(\bar{A}_{B_{\rho}(0)}(t, \nabla v)\right)=0 \quad \text { in } \tilde{\Omega}_{\rho}(0), \\
v=w \text { on } \partial_{p} \tilde{\Omega}_{\rho}(0),
\end{array}\right.
$$

where $\tilde{\Omega}_{\rho}(0)=\left(\Omega \cap B_{\rho}(0)\right) \times\left(t_{0}-\rho^{2}, t_{0}\right)\left(0<t_{0}<T\right)$. We put $v=w$ outside $\tilde{\Omega}_{\rho}(0)$. As Lemma 2.3 we have the following result.

Lemma 2.7 Let $\theta_{2}$ be the constant in Lemma 2.5. There exist positive constants $C_{1}=$ $C_{1}\left(N, \Lambda_{1}, \Lambda_{2}\right)$ and $C_{2}=C_{2}\left(\Lambda_{1}, \Lambda_{2}\right)$ such that

$$
f_{Q_{\rho}\left(0, t_{0}\right)}|\nabla w-\nabla v|^{2} d x d t \leq C_{1}\left([A]_{S_{2}}^{R}\right)^{2} f_{Q_{\rho}\left(0, t_{0}\right)}|\nabla w|^{2} d x d t,
$$

with $s_{1}=\frac{2 \theta_{2}}{\theta_{2}-2}$ and

$$
C_{2}^{-1} \int_{Q_{\rho}\left(0, t_{0}\right)}|\nabla v|^{2} d x d t \leq \int_{Q_{\rho}\left(0, t_{0}\right)}|\nabla w|^{2} d x d t \leq C_{2} \int_{Q_{\rho}\left(0, t_{0}\right)}|\nabla v|^{2} d x d t .
$$

We can see that if the boundary of $\Omega$ is irregular enough, then the $L^{\infty}$-norm of $\nabla v$ up to $\partial \Omega \cap B_{\rho}(0) \times\left(t_{0}-\rho^{2}, t_{0}\right)$ may not exist. For our purpose, we will consider another equation:

$$
\left\{\begin{array}{l}
V_{t}-\operatorname{div}\left(\bar{A}_{B_{\rho}(0)}(t, \nabla V)\right)=0 \quad \text { in } Q_{\rho}^{+}\left(0, t_{0}\right) \\
V=0 \text { on } T_{\rho}\left(0, t_{0}\right)
\end{array}\right.
$$

where $Q_{\rho}^{+}\left(0, t_{0}\right)=B_{\rho}^{+}(0) \times\left(t_{0}-\rho^{2}, t_{0}\right)$ and $T_{\rho}\left(0, t_{0}\right)=Q_{\rho}\left(0, t_{0}\right) \cap\left\{x_{N}=0\right\}$.

A weak solution $V$ of above problem is understood in the following sense: the zero extension of $V$ to $Q_{\rho}\left(0, t_{0}\right)$ is in $C\left(t_{0}-\rho^{2}, t_{0} ; L^{2}\left(B_{\rho}(0)\right)\right) \cap L_{\text {loc }}^{2}\left(t_{0}-\rho^{2}, t_{0} ; H^{1}\left(B_{\rho}(0)\right)\right)$ and for every $\varphi \in C_{c}^{1}\left(Q_{\rho}^{+}\left(0, t_{0}\right)\right)$ there holds

$$
-\int_{Q_{\rho}^{+}\left(0, t_{0}\right)} V \varphi_{t} d x d t+\int_{Q_{\rho}^{+}\left(0, t_{0}\right)} \bar{A}_{B_{\rho}(0)}(t, \nabla V) \nabla \varphi d x d t=0 .
$$

We have the following $L^{\infty}$ gradient estimate up to the boundary for $V$. The following Lemma was obtained in [19, Lemma 7.12]. 
Lemma 2.8 For any $\varepsilon>0$ there exists a small $\delta_{0}=\delta_{0}\left(N, \Lambda_{1}, \Lambda_{2}, \varepsilon\right) \in(0,1 / 2)$ such that if $v \in C\left(t_{0}-\rho^{2}, t_{0} ; L^{2}\left(\Omega \cap B_{\rho}(0)\right)\right) \cap L^{2}\left(t_{0}-\rho^{2}, t_{0} ; H^{1}\left(\Omega \cap B_{\rho}(0)\right)\right)$ is a solution of (2.14) and under condition (2.13) with $\delta \in\left(0, \delta_{0}\right)$, there exists a weak solution $V \in$ $C\left(t_{0}-\rho^{2}, t_{0} ; L^{2}\left(B_{\rho}^{+}(0)\right)\right) \cap L^{2}\left(t_{0}-\rho^{2}, t_{0} ; H^{1}\left(B_{\rho}^{+}(0)\right)\right)$ of (2.17), whose zero extension to $Q_{\rho}\left(0, t_{0}\right)$ satisfies

$$
\begin{aligned}
& \|\nabla V\|_{L^{\infty}\left(Q_{\rho / 4}\left(0, t_{0}\right)\right)}^{2} \leq C f_{Q_{\rho}\left(0, t_{0}\right)}|\nabla v|^{2} d x d t \\
& f_{Q_{\rho / 8}\left(0, t_{0}\right)}|\nabla v-\nabla V|^{2} d x d t \leq \varepsilon^{2} f_{Q_{\rho}\left(0, t_{0}\right)}|\nabla v|^{2} d x d t,
\end{aligned}
$$

for some $C=C\left(N, \Lambda_{1}, \Lambda_{2}\right)>0$.

Theorem 2.9 Let $s_{2}$ be as in Lemma 2.7. For any $\varepsilon>0$ there exists a small $\delta_{0}=$ $\delta_{0}\left(N, \Lambda_{1}, \Lambda_{2}, \varepsilon\right) \in(0,1 / 2)$ such that the following holds. If $\Omega$ is a $\left(\delta, R_{0}\right)$-Reifenberg flat domain with $\delta \in\left(0, \delta_{0}\right)$, there is a function $V \in L^{2}\left(t_{0}-(R / 9)^{2}, t_{0} ; H^{1}\left(B_{R / 9}\left(x_{0}\right)\right)\right) \cap$ $L^{\infty}\left(t_{0}-(R / 9)^{2}, t_{0} ; W^{1, \infty}\left(B_{R / 9}\left(x_{0}\right)\right)\right)$ such that

$$
\|\nabla V\|_{L^{\infty}\left(Q_{R / 9}\left(x_{0}, t_{0}\right)\right)}^{2} \leq C f_{Q_{6 R}\left(x_{0}, t_{0}\right)}|\nabla u|^{2} d x d t+C f_{Q_{6 R}\left(x_{0}, t_{0}\right)}|F|^{2} d x d t
$$

and

$$
\begin{aligned}
& f_{Q_{R / 9}\left(x_{0}, t_{0}\right)}|\nabla u-\nabla V|^{2} d x d t \\
& \quad \leq C\left(\varepsilon^{2}+\left([A]_{S_{2}}^{R_{0}}\right)^{2}\right) f_{Q_{6 R}\left(x_{0}, t_{0}\right)}|\nabla u|^{2} d x d t+C\left(\varepsilon^{2}+1+\left([A]_{S_{2}}^{R_{0}}\right)^{2}\right) f_{Q_{6 R}\left(x_{0}, t_{0}\right)}|F|^{2} d x d t,
\end{aligned}
$$

for some $C=C\left(N, \Lambda_{1}, \Lambda_{2}\right)>0$.

Proof Let $x_{0} \in \partial \Omega, 0<t_{0}<T$ and $\rho=R(1-\delta)$, we may assume that $0 \in \Omega, x_{0}=$ $(0, \ldots,-\delta \rho /(1-\delta))$ and

$$
B_{\rho}^{+}(0) \subset \Omega \cap B_{\rho}(0) \subset B_{\rho}(0) \cap\left\{x_{N}>-4 \rho \delta\right\} .
$$

We have also

$$
Q_{R / 9}\left(x_{0}, t_{0}\right) \subset Q_{\rho / 8}\left(0, t_{0}\right) \subset Q_{\rho / 4}\left(0, t_{0}\right) \subset Q_{\rho}\left(0, t_{0}\right) \subset Q_{6 \rho}\left(0, t_{0}\right) \subset Q_{6 R}\left(x_{0}, t_{0}\right),
$$

provided that $0<\delta<1 / 625$. Let $w$ and $v$ be as in Lemmas 2.6 and 2.7. By Lemma 2.8 for any $\varepsilon>0$ we can find a small positive $\delta=\delta\left(N, \Lambda_{1}, \Lambda_{2}, \varepsilon\right)<1 / 625$ such that there is a function $V \in L^{2}\left(t_{0}-\rho^{2}, t_{0} ; H^{1}\left(B_{\rho}(0)\right)\right) \cap L^{\infty}\left(t_{0}-\rho^{2}, t_{0} ; W^{1, \infty}\left(B_{\rho}(0)\right)\right)$ satisfying

$$
\begin{aligned}
\|\nabla V\|_{L^{\infty}\left(Q_{\rho / 4}\left(0, t_{0}\right)\right)}^{2} & \leq c_{1} f_{Q_{\rho}\left(0, t_{0}\right)}|\nabla v|^{2} d x d t, \\
f_{Q_{\rho / 8}\left(0, t_{0}\right)}|\nabla v-\nabla V|^{2} & \leq \varepsilon^{2} f_{Q_{\rho}\left(0, t_{0}\right)}|\nabla v|^{2} d x d t .
\end{aligned}
$$


Then, by (2.16) in Lemma 2.7 and (2.21) we get

$$
\begin{aligned}
\|\nabla V\|_{L^{\infty}\left(Q_{R / 9}\left(x_{0}, t_{0}\right)\right)}^{2} & \leq c_{2} f_{Q_{\rho}\left(0, t_{0}\right)}|\nabla w|^{2} d x d t \\
& \leq c_{3} f_{Q_{6 R}\left(x_{0}, t_{0}\right)}|\nabla w|^{2} d x d t,
\end{aligned}
$$

and

$$
f_{Q_{R / 9}\left(x_{0}, t_{0}\right)}|\nabla v-\nabla V|^{2} d x d t \leq c_{4} \varepsilon^{2} f_{Q_{6 R}\left(x_{0}, t_{0}\right)}|\nabla w|^{2} d x d t .
$$

Therefore, from (2.12) in Lemma 2.6 and (2.22) we get (2.18).

Next we prove (2.19). Since (2.21), we have

$$
\begin{aligned}
f_{Q_{R / 9}\left(x_{0}, t_{0}\right)}|\nabla u-\nabla V|^{2} d x d t \leq & c_{5} f_{Q_{\rho / 8}\left(0, t_{0}\right)}|\nabla u-\nabla V|^{2} d x d t \\
\leq & c_{6} f_{Q_{\rho / 8}\left(0, t_{0}\right)}|\nabla u-\nabla w|^{2} d x d t \\
& +c_{6} f_{Q_{\rho / 8}\left(0, t_{0}\right)}|\nabla w-\nabla v|^{2} d x d t \\
& +c_{6} f_{Q_{\rho / 8}\left(0, t_{0}\right)}|\nabla v-\nabla V|^{2} d x d t .
\end{aligned}
$$

Using (2.12) in Lemma 2.6 and (2.15), (2.16) in Lemma 2.7 and (2.23) we find that

$$
\begin{aligned}
f_{Q_{\rho / 8}\left(0, t_{0}\right)}|\nabla u-\nabla w|^{2} d x d t & \leq c_{6} f_{Q_{6 R}\left(x_{0}, t_{0}\right)}|F|^{2} d x d t, \\
f_{Q_{\rho / 8}\left(0, t_{0}\right)}|\nabla v-\nabla w|^{2} d x d t & \leq c_{7}\left([A]_{S_{2}}^{R_{0}}\right)^{2} f_{Q_{6 R}\left(0, t_{0}\right)}|\nabla w|^{2} d x d t \\
& \leq c_{8}\left([A]_{S_{2}}^{R_{0}}\right)^{2}\left(f_{Q_{6 R}\left(x_{0}, t_{0}\right)}|\nabla u|^{2} d x d t+f_{Q_{6 R}\left(x_{0}, t_{0}\right)}|F|^{2} d x d t\right),
\end{aligned}
$$

and

$$
f_{Q_{\rho / 8}\left(0, t_{0}\right)}|\nabla v-\nabla V|^{2} d x d t \leq c_{9} \varepsilon^{2}\left(f_{Q_{6 R}\left(x_{0}, t_{0}\right)}|\nabla u|^{2} d x d t+f_{Q_{6 R}\left(x_{0}, t_{0}\right)}|F|^{2} d x d t\right) .
$$

Then we derive (2.19). This completes the proof.

\section{Global integral gradient bounds for parabolic equations}

The following good- $\lambda$ type estimate will be essential for our global estimates later.

Theorem 3.1 Let $s_{1}, s_{2}$ be as in Lemma 2.3, 2.7 and $s_{0}=\max \left\{s_{1}, s_{2}\right\}$. Let $w \in \mathbf{A}_{\infty}$, $F \in L^{2}\left(\Omega_{T}, \mathbb{R}^{N}\right)$. Let $u \in L^{2}\left(0, T ; H_{0}^{1}(\Omega)\right)$ be the weak solution to equation (1.1) in $\Omega_{T}$. For any $\varepsilon>0, R_{0}>0$ one finds $\delta_{1}=\delta_{1}\left(N, \Lambda_{1}, \Lambda_{2}, \varepsilon,[w]_{\mathbf{A}_{\infty}}\right) \in(0,1 / 2)$ and $\delta_{2}=\delta_{2}\left(N, \Lambda_{1}, \Lambda_{2}, \varepsilon,[w]_{\mathbf{A}_{\infty}}, T_{0} / R_{0}\right) \in(0,1)$ and $\Lambda=\Lambda\left(N, \Lambda_{1}, \Lambda_{2}\right)>0$ such that if $\Omega$ is a $\left(\delta_{1}, R_{0}\right)$-Reifenberg flat domain and $[A]_{s_{0}}^{R_{0}} \leq \delta_{1}$ then

$$
w\left(\left\{\mathcal{M}\left(|\nabla u|^{2}\right)>\Lambda \lambda, \mathcal{M}\left(|F|^{2}\right) \leq \delta_{2} \lambda\right\} \cap \Omega_{T}\right) \leq B \varepsilon w\left(\left\{\mathcal{M}\left(|\nabla u|^{2}\right)>\lambda\right\} \cap \Omega_{T}\right)
$$


for all $\lambda>0$, where the constant $B$ depends only on $N, \Lambda_{1}, \Lambda_{2}, T_{0} / R_{0},[w]_{\mathbf{A}_{\infty}}$.

To prove above estimate, we will use L. Caddarelli and I. Peral's technique in [5]. Namely, it is based on the following technical lemma whose proof is a consequence of Lebesgue Differentiation Theorem and the standard Vitali covering lemma, can be found in $[3,15]$ with some modifications to fit the setting here.

Lemma 3.2 Let $\Omega$ be a $\left(\delta, R_{0}\right)$-Reifenberg flat domain with $\delta<1 / 4$ and let $w$ be an $\mathbf{A}_{\infty}$ weight. Suppose that the sequence of balls $\left\{B_{r}\left(y_{i}\right)\right\}_{i=1}^{L}$ with centers $y_{i} \in \bar{\Omega}$ and radius $r \leq R_{0} / 4$ covers $\Omega$. Set $s_{i}=T-i r^{2} / 2$ for all $i=0,1, \ldots,\left[\frac{2 T}{r^{2}}\right]$. Let $E \subset F \subset \Omega_{T}$ be measurable sets for which there exists $0<\varepsilon<1$ such that $w(E)<\varepsilon w\left(\tilde{Q}_{r}\left(y_{i}, s_{j}\right)\right)$ for all $i=1, \ldots, L, j=0,1, \ldots,\left[\frac{2 T}{r^{2}}\right]$; and for all $(x, t) \in \Omega_{T}, \rho \in(0,2 r]$, we have $\tilde{Q}_{\rho}(x, t) \cap \Omega_{T} \subset F$ if $w\left(E \cap \tilde{Q}_{\rho}(x, t)\right) \geq \varepsilon w\left(\tilde{Q}_{\rho}(x, t)\right)$. Then $w(E) \leq \varepsilon B w(F)$ for $a$ constant $B$ depending only on $N$ and $[w]_{\mathbf{A}_{\infty}}$.

Proof Note that $[A]_{s_{1}}^{R_{0}},[A]_{s_{2}}^{R_{0}} \leq[A]_{s_{0}}^{R_{0}}$. Let $\varepsilon \in(0,1)$. Set $E_{\lambda, \delta_{2}}=\left\{\mathcal{M}\left(|\nabla u|^{2}\right)>\right.$ $\left.\Lambda \lambda, \mathcal{M}\left(|F|^{2}\right) \leq \delta_{2} \lambda\right\} \cap \Omega_{T}$ and $F_{\lambda}=\left\{\mathcal{M}\left(|\nabla u|^{2}\right)>\lambda\right\} \cap \Omega_{T}$ for $\delta_{2} \in(0,1), \Lambda>0$ and $\lambda>0$. Let $\left\{y_{i}\right\}_{i=1}^{L} \subset \Omega$ and a ball $B_{0}$ with radius $2 T_{0}$ such that

$$
\Omega \subset \bigcup_{i=1}^{L} B_{r_{0}}\left(y_{i}\right) \subset B_{0},
$$

where $r_{0}=\min \left\{R_{0} / 1080, T_{0}\right\}$. Let $s_{j}=T-j r_{0}^{2} / 2$ for all $j=0,1, \ldots,\left[\frac{2 T}{r_{0}^{2}}\right]$ and $Q_{2 T_{0}}=$ $B_{0} \times\left(T-4 T_{0}^{2}, T\right)$. So,

$$
\Omega_{T} \subset \bigcup_{i, j} Q_{r_{0}}\left(y_{i}, s_{j}\right) \subset Q_{2 T_{0}}
$$

We verify that

$$
w\left(E_{\lambda, \delta_{2}}\right) \leq \varepsilon w\left(\tilde{Q}_{r_{0}}\left(y_{i}, s_{j}\right)\right) \quad \forall \lambda>0
$$

for some $\delta_{2}$ small enough depending on $n, p, \alpha, \beta, \epsilon,[w]_{\mathbf{A}_{\infty}}, T_{0} / R_{0}$.

In fact, we can assume that $E_{\lambda, \delta_{2}} \neq \varnothing$ so $\int_{\Omega_{T}}|F|^{2} d x d t \leq c_{1}\left|Q_{2 T_{0}}\right| \delta_{2} \lambda$. Recalling that $\mathcal{M}$ is a bounded operator from $L^{1}\left(\mathbb{R}^{N+1}\right)$ into $L^{1, \infty}\left(\mathbb{R}^{N+1}\right)$, we find

$$
\left|E_{\lambda, \delta_{2}}\right| \leq \frac{c_{2}}{\Lambda \lambda} \int_{\Omega_{T}}|\nabla u|^{2} d x d t
$$

Using (2.1) in Proposition 1, we get

$$
\begin{aligned}
\left|E_{\lambda, \delta_{2}}\right| & \leq \frac{c_{3}}{\Lambda \lambda} \int_{\Omega_{T}}|F|^{2} d x d t \\
& \leq c_{4} \delta_{2}\left|Q_{2 T_{0}}\right|,
\end{aligned}
$$

which implies

$$
w\left(E_{\lambda, \delta_{2}}\right) \leq C\left(\frac{\left|E_{\lambda, \delta_{2}}\right|}{\left|Q_{2 T_{0}}\right|}\right)^{v} w\left(Q_{2 T_{0}}\right) \leq C\left(c_{4} \delta_{2}\right)^{v} w\left(Q_{2 T_{0}}\right),
$$


where $(C, v)=[w]_{\mathbf{A}_{\infty}}$. It is well-known that (see, e.g [7]) there exist $C_{1}=C_{1}(N, C, v)$ and $v_{1}=v_{1}(N, C, v)$ such that

$$
\frac{w\left(\tilde{Q}_{2 T_{0}}\right)}{w\left(\tilde{Q}_{r_{0}}\left(y_{i}, s_{j}\right)\right)} \leq C_{1}\left(\frac{\left|\tilde{Q}_{2 T_{0}}\right|}{\left|\tilde{Q}_{r_{0}}\left(y_{i}, s_{j}\right)\right|}\right)^{v_{1}} \forall i, j
$$

Therefore,

$$
w\left(E_{\lambda, \delta_{2}}\right) \leq C\left(c_{4} \delta_{2}\right)^{v} C_{1}\left(\frac{\left|\tilde{Q}_{T_{0}}\right|}{\left|\tilde{Q}_{r_{0}}\left(y_{i}, s_{j}\right)\right|}\right)^{v_{1}} w\left(\tilde{Q}_{r_{0}}\left(y_{i}, s_{j}\right)\right)<\varepsilon w\left(\tilde{Q}_{r_{0}}\left(y_{i}, s_{j}\right)\right) \quad \forall i, j,
$$

where $\delta_{2} \leq \varepsilon^{1 / \nu}\left(2 C C_{1} c_{4}^{\nu}\left(T_{0} r_{0}^{-1}\right)^{(N+2) v_{1}}\right)^{-1 / v}$. Thus (3.2) follows.

Next we verify that for all $(x, t) \in \Omega_{T}, r \in\left(0,2 r_{0}\right]$ and $\lambda>0$ we have $\tilde{Q}_{r}(x, t) \cap \Omega_{T} \subset F_{\lambda}$ provided

$$
w\left(E_{\lambda, \delta_{2}} \cap \tilde{Q}_{r}(x, t)\right) \geq \varepsilon w\left(Q_{r}(x, t)\right),
$$

for some $\delta_{2} \leq \min \left\{1, \varepsilon^{1 / \nu}\left(2 C C_{1} c_{4}^{\nu}\left(T_{0} r_{0}^{-1}\right)^{(N+2) v_{1}}\right)^{-1 / v}\right\}$. Indeed, take $(x, t) \in \Omega_{T}$ and $0<$ $r \leq 2 r_{0}$. Now assume that $\tilde{Q}_{r}(x, t) \cap \Omega_{T} \cap F_{\lambda}^{c} \neq \emptyset$ and $E_{\lambda, \delta_{2}} \cap \tilde{Q}_{r}(x, t) \neq \emptyset$ i.e, there exist $\left(x_{1}, t_{1}\right),\left(x_{2}, t_{2}\right) \in \tilde{Q}_{r}(x, t) \cap \Omega_{T}$ such that $\mathcal{M}\left(|\nabla u|^{2}\right)\left(x_{1}, t_{1}\right) \leq \lambda$ and $\mathcal{M}\left(|F|^{2}\right)\left(x_{2}, t_{2}\right) \leq$ $\delta_{2} \lambda$. We need to prove that

$$
\left.w\left(E_{\lambda, \delta_{2}} \cap \tilde{Q}_{r}(x, t)\right)\right)<\varepsilon w\left(\tilde{Q}_{r}(x, t)\right) .
$$

Using $\mathcal{M}\left(|\nabla u|^{2}\right)\left(x_{1}, t_{1}\right) \leq \lambda$, we can see that

$$
\mathcal{M}\left(|\nabla u|^{2}\right)(y, s) \leq \max \left\{\mathcal{M}\left(\chi_{\tilde{Q}_{2 r}(x, t)}|\nabla u|^{2}\right)(y, s), 3^{N+2} \lambda\right\} \quad \forall(y, s) \in \tilde{Q}_{r}(x, t) .
$$

Therefore, for all $\lambda>0$ and $\Lambda \geq 3^{N+2}$,

$$
E_{\lambda, \delta_{2}} \cap \tilde{Q}_{r}(x, t)=\left\{\mathcal{M}\left(\chi_{\tilde{Q}_{2 r}(x, t)}|\nabla u|^{2}\right)>\Lambda \lambda, \mathcal{M}\left(|F|^{2}\right) \leq \delta_{2} \lambda\right\} \cap \Omega_{T} \cap \tilde{Q}_{r}(x, t) .
$$

In particular, $E_{\lambda, \delta_{2}} \cap \tilde{Q}_{r}(x, t)=\emptyset$ if $\bar{B}_{8 r}(x) \subset \subset \mathbb{R}^{N} \backslash \Omega$. Thus, it is enough to consider the case $B_{8 r}(x) \subset \subset \Omega$ and the case $B_{8 r}(x) \cap \Omega \neq \emptyset$.

First assume $B_{8 r}(x) \subset \subset \Omega$. Let $v$ be as in Theorem 2.4 with $Q_{2 R}=Q_{8 r}\left(x, t_{0}\right)$ and $t_{0}=\min \left\{t+2 r^{2}, T\right\}$. We have

$$
\|\nabla v\|_{L^{\infty}\left(Q_{2 r}\left(x, t_{0}\right)\right)}^{2} \leq c_{5} f_{Q_{8 r}\left(x, t_{0}\right)}|\nabla u|^{2} d x d t+c_{5} f_{Q_{8 r}\left(x, t_{0}\right)}|F|^{2} d x d t,
$$

and

$$
\begin{aligned}
f_{Q_{4 r}\left(x, t_{0}\right)}|\nabla u-\nabla v|^{2} d x d t \leq & c_{5} f_{Q_{8 r}\left(x, t_{0}\right)}|F|^{2} d x d t \\
& +c_{5}\left([A]_{s_{1}}^{R}\right)^{2}\left(f_{Q_{8 r}\left(x, t_{0}\right)}|\nabla u|^{2} d x d t+f_{Q_{8 r}\left(x, t_{0}\right)}|F|^{2} d x d t\right) .
\end{aligned}
$$


Thanks to $\mathcal{M}\left(|\nabla u|^{2}\right)\left(x_{1}, t_{1}\right) \leq \lambda$ and $\mathcal{M}\left(|F|^{2}\right)\left(x_{2}, t_{2}\right) \leq \delta_{2} \lambda$ with $\left(x_{1}, t_{1}\right),\left(x_{2}, t_{2}\right) \in$ $Q_{r}(x, t)$, we find $Q_{8 r}\left(x, t_{0}\right) \subset \tilde{Q}_{17 r}\left(x_{1}, t_{1}\right), \tilde{Q}_{17 r}\left(x_{2}, t_{2}\right)$ and

$$
\begin{aligned}
\|\nabla v\|_{L^{\infty}\left(Q_{2 r}\left(x, t_{0}\right)\right)}^{2} & \leq c_{6} f_{\tilde{Q}_{17 r}\left(x_{1}, t_{1}\right)}|\nabla u|^{2} d x d t+c_{6} f_{\tilde{Q}_{17 r}\left(x_{2}, t_{2}\right)}|F|^{2} d x d t \\
& \leq c_{6}\left(1+\delta_{2}\right) \lambda \\
& \leq c_{7} \lambda,
\end{aligned}
$$

and

$$
\begin{aligned}
f_{Q_{4 r}\left(x, t_{0}\right)}|\nabla u-\nabla v|^{2} d x d t & \leq c_{8} \delta_{2} \lambda+c_{5}\left([A]_{S_{0}}^{R}\right)^{2}\left(1+\delta_{2}\right) \lambda \\
& \leq c_{9}\left(\delta_{2}+\delta_{1}^{2}\left(1+\delta_{2}\right)\right) \lambda
\end{aligned}
$$

Here we used $[A]_{s_{0}}^{R_{0}} \leq \delta_{1}$ in the last inequality.

In view of (3.6) we see that for $\Lambda \geq \max \left\{3^{N+2}, 4 c_{7}\right\}$,

$$
\left|\left\{\mathcal{M}\left(\chi_{\tilde{Q}_{2 r}(x, t)}|\nabla v|^{2}\right)>\Lambda \lambda / 4\right\} \cap \tilde{Q}_{r}(x, t)\right|=0 .
$$

Leads to

$$
\left|E_{\lambda, \delta_{2}} \cap \tilde{Q}_{r}(x, t)\right| \leq\left|\left\{\mathcal{M}\left(\chi_{\tilde{Q}_{2 r}(x, t)}|\nabla u-\nabla v|^{2}\right)>\Lambda \lambda / 4\right\} \cap \tilde{Q}_{r}(x, t)\right| .
$$

Therefore, by bound of operator $\mathcal{M}$ from $L^{1}\left(\mathbb{R}^{N+1}\right)$ to $L^{1, \infty}\left(\mathbb{R}^{N+1}\right)$ and (3.7), $\tilde{Q}_{2 r}(x, t) \subset$ $Q_{4 r}\left(x, t_{0}\right)$ we deduce

$$
\begin{aligned}
\left|E_{\lambda, \delta_{2}} \cap \tilde{Q}_{r}(x, t)\right| & \leq \frac{c_{10}}{\lambda} \int_{\tilde{Q}_{2 r}(x, t)}|\nabla u-\nabla v|^{2} d x d t \\
& \leq c_{11}\left(\delta_{2}+\delta_{1}^{2}\left(1+\delta_{2}\right)\right)\left|Q_{r}(x, t)\right| .
\end{aligned}
$$

Thus,

$$
\begin{aligned}
w\left(E_{\lambda, \delta_{2}} \cap \tilde{Q}_{r}(x, t)\right) & \leq C\left(\frac{\left|E_{\lambda, \delta_{2}} \cap \tilde{Q}_{r}(x, t)\right|}{\left|\tilde{Q}_{r}(x, t)\right|}\right)^{v} w\left(\tilde{Q}_{r}(x, t)\right) \\
& \leq C\left(c_{11}\left(\delta_{2}+\delta_{1}^{2}\left(1+\delta_{2}\right)\right)\right)^{v} w\left(\tilde{Q}_{r}(x, t)\right) \\
& <\varepsilon w\left(\tilde{Q}_{r}(x, t)\right) .
\end{aligned}
$$

where $\delta_{2}, \delta_{1}$ are appropriately chosen and $(C, v)=[w]_{\mathbf{A}_{\infty}}$.

Next assume $B_{8 r}(x) \cap \Omega \neq \emptyset$. Let $x_{3} \in \partial \Omega$ such that $\left|x_{3}-x\right|=\operatorname{dist}(x, \partial \Omega)$. Set $t_{0}=\min \left\{t+2 r^{2}, T\right\}$. We have

$$
\begin{aligned}
& Q_{2 r}\left(x, t_{0}\right) \subset Q_{10 r}\left(x_{3}, t_{0}\right) \subset Q_{540 r}\left(x_{3}, t_{0}\right) \subset \tilde{Q}_{1080 r}\left(x_{3}, t\right) \subset \tilde{Q}_{1088 r}(x, t) \\
& \quad \subset \tilde{Q}_{1089 r}\left(x_{1}, t_{1}\right),
\end{aligned}
$$

and

$$
Q_{540 r}\left(x_{3}, t_{0}\right) \subset \tilde{Q}_{1080 r}\left(x_{3}, t\right) \subset \tilde{Q}_{1088 r}(x, t) \subset \tilde{Q}_{1089 r}\left(x_{2}, t_{2}\right) .
$$

Let $V$ be as in Theorem 2.9 with $Q_{6 R}=Q_{540 r}\left(x_{3}, t_{0}\right)$ and $\varepsilon=\delta_{3} \in(0,1)$. We have

$$
\|\nabla V\|_{L^{\infty}\left(Q_{10 r}\left(x_{3}, t_{0}\right)\right)}^{2} \leq c_{12} f_{Q_{540 r}\left(x_{3}, t_{0}\right)}|\nabla u|^{2} d x d t+c_{12} f_{Q_{540 r}\left(x_{3}, t_{0}\right)}|F|^{2} d x d t,
$$


and

$$
\begin{aligned}
& f_{Q_{10 r}\left(x_{3}, t_{0}\right)}|\nabla u-\nabla V|^{2} d x d t \\
& \quad \leq c_{12}\left(\delta_{3}^{2}+\left([A]_{S_{2}}^{R_{0}}\right)^{2}\right) f_{Q_{540 r}\left(x_{3}, t_{0}\right)}|\nabla u|^{2} d x d t+c_{12}\left(\delta_{3}^{2}+1+\left([A]_{S_{2}}^{R_{0}}\right)^{2}\right) \\
& f_{Q_{540 r}\left(x_{3}, t_{0}\right)}|F|^{2} d x d t .
\end{aligned}
$$

Since $\mathcal{M}\left(|\nabla u|^{2}\right)\left(x_{1}, t_{1}\right) \leq \lambda, \mathcal{M}\left(|F|^{2}\right)\left(x_{2}, t_{2}\right) \leq \delta_{2} \lambda$ and (3.8), (3.9) we get

$$
\begin{aligned}
\|\nabla V\|_{L^{\infty}\left(Q_{10 r}\left(x_{3}, t_{0}\right)\right)}^{2} & \leq c_{13} f_{\tilde{Q}_{1089 r}\left(x_{1}, t_{1}\right)}|\nabla u|^{2} d x d t+c_{13} f_{\tilde{Q}_{1089 r}\left(x_{1}, t_{1}\right)}|F|^{2} d x d t \\
& \leq c_{14}\left(1+\delta_{2}\right) \lambda \\
& \leq c_{15} \lambda,
\end{aligned}
$$

and

$$
\begin{aligned}
f_{Q_{10 r}\left(x_{3}, t_{0}\right)}|\nabla u-\nabla V|^{2} d x d t & \leq c_{16}\left(\left(\delta_{3}^{2}+\left([A]_{S_{2}}^{R_{0}}\right)^{2}\right)+\left(\delta_{3}^{2}+1+\left([A]_{S_{2}}^{R_{0}}\right)^{2}\right) \delta_{2}\right) \lambda \\
& \leq c_{16}\left(\left(\delta_{3}^{2}+\delta_{1}^{2}\right)+\left(\delta_{3}^{2}+1+\delta_{1}^{2}\right) \delta_{2}\right) \lambda
\end{aligned}
$$

Notice that we have used $[A]_{s_{0}}^{R_{0}} \leq \delta_{1}$ in the last inequality.

Now set $\Lambda=\max \left\{3^{N+2}, 4 c_{7}, 4 c_{15}\right\}$. As above we also have

$$
\left|E_{\lambda, \delta_{2}} \cap \tilde{Q}_{r}(x, t)\right| \leq\left|\left\{\mathcal{M}\left(\chi_{\tilde{Q}_{2 r}(x, t)}|\nabla u-\nabla V|^{2}\right)>\Lambda \lambda / 4\right\} \cap \tilde{Q}_{r}(x, t)\right| .
$$

Therefore using (3.10) we obtain

$$
\begin{aligned}
\left|E_{\lambda, \delta_{2}} \cap \tilde{Q}_{r}(x, t)\right| & \leq \frac{c_{17}}{\lambda} \int_{\tilde{Q}_{2 r}(x, t)}|\nabla u-\nabla V|^{2} d x d t \\
& \leq c_{18}\left(\left(\delta_{3}^{2}+\delta_{1}^{2}\right)+\left(\delta_{3}^{2}+1+\delta_{1}^{2}\right) \delta_{2}\right)\left|\tilde{Q}_{r}(x, t)\right| .
\end{aligned}
$$

Thus

$$
\begin{aligned}
w\left(E_{\lambda, \delta_{2}} \cap \tilde{Q}_{r}(x, t)\right) & \leq C\left(\frac{\left|E_{\lambda, \delta_{2}} \cap \tilde{Q}_{r}(x, t)\right|}{\left|\tilde{Q}_{r}(x, t)\right|}\right)^{v} w\left(\tilde{Q}_{r}(x, t)\right) \\
& \leq C\left(c_{18}\left(\left(\delta_{3}^{2}+\delta_{1}^{2}\right)+\left(\delta_{3}^{2}+1+\delta_{1}^{2}\right) \delta_{2}\right)\right)^{v} w\left(\tilde{Q}_{r}(x, t)\right) \\
& <\varepsilon w\left(\tilde{Q}_{r}(x, t)\right),
\end{aligned}
$$

where $\delta_{3}, \delta_{1}, \delta_{2}$ are appropriately chosen and $(C, v)=[w]_{\mathbf{A}_{\infty}}$.

Therefore, for all $(x, t) \in \Omega_{T}, r \in\left(0,2 r_{0}\right]$ and $\lambda>0$, if

$$
w\left(E_{\lambda, \delta_{2}} \cap \tilde{Q}_{r}(x, t)\right) \geq \varepsilon w\left(\tilde{Q}_{r}(x, t)\right),
$$

then

$$
\tilde{Q}_{r}(x, t) \cap \Omega_{T} \subset F_{\lambda},
$$

where $\delta_{1}=\delta_{1}\left(N, \Lambda_{1}, \Lambda_{2}, \varepsilon,[w]_{\mathbf{A}_{\infty}}\right) \in(0,1)$ and $\delta_{2}=\delta_{2}\left(N, \Lambda_{1}, \Lambda_{2}, \varepsilon,[w]_{\mathbf{A}_{\infty}}, T_{0} / R_{0}\right) \in$ $(0,1)$. Combining this with (3.2), we can apply Lemma 3.2 to get the result. 
Proof of Theorem 1.1 By Theorem 3.1, for any $\varepsilon>0, R_{0}>0$ one finds $\delta=$ $\delta_{1}\left(N, \Lambda_{1}, \Lambda_{2}, \varepsilon,[w]_{\mathbf{A}_{\infty}}\right) \in(0,1 / 2)$ and $\delta_{2}=\delta_{2}\left(N, \Lambda_{1}, \Lambda_{2}, \varepsilon,[w]_{\mathbf{A}_{\infty}}, T_{0} / R_{0}\right) \in(0,1)$ and $\Lambda=\Lambda\left(N, \Lambda_{1}, \Lambda_{2}\right)>0, s_{0}=s_{0}\left(N, \Lambda_{1}, \Lambda_{2}\right)$ such that if $\Omega$ is a $\left(\delta, R_{0}\right)$ - Reifenberg flat domain and $[A]_{s_{0}}^{R_{0}} \leq \delta$ then

$$
w\left(\left\{\mathcal{M}\left(|\nabla u|^{2}\right)>\Lambda \lambda, \mathcal{M}\left[|F|^{2}\right] \leq \delta_{2} \lambda\right\} \cap \Omega_{T}\right) \leq B \varepsilon w\left(\left\{\mathcal{M}\left(|\nabla u|^{2}\right)>\lambda\right\} \cap \Omega_{T}\right),
$$

for all $\lambda>0$, where the constant $B$ depends only on $N, \Lambda_{1}, \Lambda_{2}, T_{0} / R_{0},[w]_{\mathbf{A}_{\infty}}$. Thus, for $s<\infty$,

$$
\begin{aligned}
\left\|\mathcal{M}\left(|\nabla u|^{2}\right)\right\|_{L_{w}^{q, s}\left(\Omega_{T}\right)}^{s}= & q \Lambda^{s} \int_{0}^{\infty} \lambda^{s}\left(w\left(\left\{\mathcal{M}\left(|\nabla u|^{2}\right)>\Lambda \lambda\right\} \cap \Omega_{T}\right)\right)^{s / q} \frac{d \lambda}{\lambda} \\
\leq & q \Lambda^{s} 2^{s / q}(B \varepsilon)^{s / q} \int_{0}^{\infty} \lambda^{s}\left(w\left(\left\{\mathcal{M}\left(|\nabla u|^{2}\right)>\lambda\right\} \cap \Omega_{T}\right)\right)^{s / q} \frac{d \lambda}{\lambda} \\
& +q \Lambda^{s} 2^{s / q} \int_{0}^{\infty} \lambda^{s}\left(w\left(\left\{\mathcal{M}\left(|F|^{2}\right)>\delta_{2} \lambda\right\} \cap \Omega_{T}\right)\right)^{s / q} \frac{d \lambda}{\lambda} \\
= & \Lambda^{s} 2^{s / q}(B \varepsilon)^{s / q} \|\left.\mathcal{M}\left(|\nabla u|^{2}\right)\right|_{L_{w}^{q, s}\left(\Omega_{T}\right)} ^{s} \\
& +\Lambda^{s} 2^{s / q} \delta_{2}^{-s}\left\|\mathcal{M}\left(|F|^{2}\right)\right\|_{L_{w}^{q, s}\left(\Omega_{T}\right)}^{s}
\end{aligned}
$$

It implies

$$
\begin{aligned}
\left\|\mathcal{M}\left(|\nabla u|^{2}\right)\right\|_{L_{w}^{q, s}\left(\Omega_{T}\right)} \leq & 2^{1 / s} \Lambda 2^{1 / q}(B \varepsilon)^{1 / q}|| \mathcal{M}\left(|\nabla u|^{2}\right) \|_{L_{w}^{q, s}\left(\Omega_{T}\right)} \\
& +2^{1 / s} \Lambda 2^{1 / q} \delta_{2}^{-1}\left\|\mathcal{M}\left(|F|^{2}\right)\right\|_{L_{w}^{q, s}\left(\Omega_{T}\right)}
\end{aligned}
$$

and this inequalities is also true when $s=\infty$.

We can choose $\varepsilon=\varepsilon(N, \Lambda, s, q, B)>0$ such that $2^{1 / s} \Lambda 2^{1 / q}(B \varepsilon)^{1 / q} \leq 1 / 2$, then we get the result.

Proof of Theorem 1.3 We recall that $A(x, t, \xi)=A(x, t) \xi$ where $A(x, t)$ is a matrix.

a. Fix $q>2,0<s \leq \infty, w \in \mathbf{A}_{q / 2}$. Assume $\|\mid F\|_{L_{w}^{q, s}\left(\Omega_{T}\right)}<\infty$. So, $F \in L^{2}\left(\Omega_{T}, \mathbb{R}^{N}\right)$ and problem (1.8) with $\mu \equiv 0, \sigma \equiv 0$ has a unique weak solution $v_{1} \in L^{2}\left(0, T, H_{0}^{1}(\Omega)\right)$. By Theorem 1.2, we find a $\delta_{1}=\delta_{1}\left(N, \Lambda_{1}, \Lambda_{2}, q, s,[w]_{\mathbf{A}_{q / 2}}\right) \in(0,1)$ such that if $\Omega$ is a $\left(\delta_{1}, R_{0}\right)$-Reifenberg flat domain and $[A]_{s_{0}}^{R_{0}} \leq \delta_{1}$ for some $R_{0}>0$ then

$$
\left\|\left|\nabla v_{1} \|\right|_{L_{w}^{q, s}\left(\Omega_{T}\right)} \leq\left. c_{1}|||F|\right|_{L_{w}^{q, s}\left(\Omega_{T}\right)},\right.
$$

where $c_{1}=c_{1}\left(N, \Lambda_{1}, \Lambda_{2}, q, s,[w]_{\mathbf{A}_{q / 2}}, T_{0} / R_{0}\right)$.

Moreover, by [19, Theorem 2.20], there exists a distribution solution $v_{2} \in$ $L^{1}\left(0, T, W_{0}^{1,1}(\Omega)\right)$ of (1.8) with $F \equiv 0$ and $\delta_{2}=\delta_{2}\left(N, \Lambda_{1}, \Lambda_{2}, q, s,[w]_{\mathbf{A}_{q / 2}}\right) \in(0,1)$ such that if $\Omega$ is a $\left(\delta_{2}, R_{0}\right)$-Reifenberg flat and $[A]_{s_{0}}^{R_{0}} \leq \delta_{2}$ for some $R_{0}>0$, then there holds

$$
\|\left.\left|\nabla v_{2}\right|\right|_{L_{w}^{q, s}\left(\Omega_{T}\right)} \leq\left. c_{2}|| \mathcal{M}_{1}[\omega]\right|_{L_{w}^{q, s}\left(\Omega_{T}\right)},
$$

where $c_{2}=c_{2}\left(N, \Lambda_{1}, \Lambda_{2}, q, s,[w]_{\mathbf{A}_{q / 2}}, T_{0} / R_{0}\right)$. In particular, $v_{2} \in L^{2}\left(0, T, H_{0}^{1}(\Omega)\right)$.

Obviously, $u:=v_{1}+v_{2}$ is a unique weak solution of (1.8) in $L^{2}\left(0, T, H_{0}^{1}(\Omega)\right)$ and from (3.12)-(3.13) we obtain (1.9) where $\Omega$ is a $\left(\delta, R_{0}\right)$-flat and $[A]_{s_{0}}^{R_{0}} \leq \delta$ with $\delta=\min \left\{\delta_{1}, \delta_{2}\right\}$. (b) Using the previous argument, we only show statement $\mathbf{b}$ in case $\mu \equiv 0, \sigma \equiv 0$. Fix $\varepsilon \in(0,1), \frac{2(\varepsilon+1)}{\varepsilon+2}<q \leq 2,0<s \leq \infty, w^{2+\varepsilon} \in \mathbf{A}_{1}$ and assume $\mathcal{M}_{1}[\omega],|F| \in L_{w}^{q, s}\left(\Omega_{T}\right)$. Set $p=\frac{2(\varepsilon+1)}{\varepsilon+2}$. 
(b.1) We prove that there is a $\delta_{3}=\delta_{3}\left(N, \Lambda_{1}, \Lambda_{2}, \varepsilon\right) \in(0,1)$ such that if $\Omega$ is a $\left(\delta, R_{0}\right)$ Reifenberg flat domain for some $R_{0}>0$, then problem (1.8) with $\mu \equiv 0, \sigma \equiv 0$ has a unique weak solution $v_{3} \in L^{p}\left(0, T, W_{0}^{1, p}(\Omega)\right)$.

Clearly, if $A^{*}(x, t, \xi)=A^{*}(x, t) \xi$, where $A^{*}(x, t)$ is the transposed matrix of $A(x, t)$ then $A^{*}$ satisfies (1.2) and (1.3) with the same constants and $\left[A^{*}\right]_{s_{0}}^{R_{0}}=[A]_{s_{0}}^{R_{0}}$.

By Theorem 1.2 there exists $\delta_{3}=\delta_{3}\left(N, \Lambda_{1}, \Lambda_{2}, \varepsilon\right) \in(0,1)$ such that if $\Omega$ is $\left(\delta_{3}, R_{0}\right)$-flat and $[A]_{s_{0}}^{R_{0}} \leq \delta_{3}$ for some $R_{0}>0$ there holds

$$
\left\|\left|\nabla \varphi \left\|_{L^{p^{\prime}\left(\Omega_{T}\right)}} \leq c_{3}|\|G\||_{L^{p^{\prime}\left(\Omega_{T}\right)}} \quad \forall G \in C^{\infty}\left(\bar{\Omega}_{T}, \mathbb{R}^{N}\right),\right.\right.\right.
$$

for some constant $c_{3}$, where $\varphi$ is a unique solution to the problem

$$
\left\{\begin{array}{l}
-\varphi_{t}-\operatorname{div}\left(A^{*}(x, t) \nabla \varphi\right)=\operatorname{div}(G) \text { in } \Omega_{T}, \\
u=0 \text { on } \partial \Omega \times(0, T) \\
u(T)=0 \text { in } \Omega
\end{array}\right.
$$

Let $F_{n} \in C_{c}^{\infty}\left(\Omega_{T}, \mathbb{R}^{N}\right)$ converge to $F$ in $L^{p}\left(\Omega_{T}, \mathbb{R}^{N}\right)$ and $u_{n}$ be a solution of problem (1.8) with $F=F_{n}$ and $\mu \equiv 0, \sigma \equiv 0$. We can choose $\varphi$ for test function,

$$
\begin{aligned}
-\int_{\Omega_{T}} \nabla u_{n} G d x d t & =-\int_{\Omega_{T}} u_{n} \varphi_{t} d x d t+\int_{\Omega_{T}} A^{*}(x, t) \nabla \varphi \nabla u_{n} d x d t \\
& =-\int_{\Omega_{T}} u_{n} \varphi_{t} d x d t+\int_{\Omega_{T}} A(x, t) \nabla u_{n} \nabla \varphi d x d t \\
& =-\int_{\Omega_{T}} \nabla \varphi F_{n} .
\end{aligned}
$$

Using Hölder inequality and (3.14) yield

$$
\left|\int_{\Omega_{T}} \nabla u_{n} G d x d t\right| \leq\left. c_{3}|||G|\right|_{L^{p^{\prime}\left(\Omega_{T}\right)}}|| \mid F_{n} \|_{L^{p}\left(\Omega_{T}\right)} \quad \forall G \in C^{\infty}\left(\bar{\Omega}_{T}, \mathbb{R}^{N}\right),
$$

it implies

$$
\left.\left\|\left|\nabla u_{n}\right|\right\|\right|_{L^{p}\left(\Omega_{T}\right)} \leq\left. c_{3}\left\||| F_{n}\right\|\right|_{L^{p}\left(\Omega_{T}\right)} .
$$

By linearity of $A$ we get

$$
\left\|\left|\nabla u_{n}-\nabla u_{m}\right|\right\|_{L^{p}\left(\Omega_{T}\right)} \leq c_{3}||\left|F_{n}-F_{m}\right| \|_{L^{p}\left(\Omega_{T}\right)} \rightarrow 0 \quad \text { as } n, m \rightarrow \infty .
$$

Thus, $u_{n}$ converges to some function $v_{3}$ in $L^{p}\left(0, T, W_{0}^{1, p}(\Omega)\right)$. Obviously, $v_{3}$ is a unique weak solution in $L^{p}\left(0, T, W_{0}^{1, p}(\Omega)\right)$ of problem (1.8) with $\mu \equiv 0, \sigma \equiv 0$. b.2. Set $\bar{w}(x, t)=$ $(w(x, t))^{-\frac{1}{p-1}}$. We have $\bar{w} \in \mathbf{A}_{p^{\prime} / 2}$ and $[\bar{w}]_{\mathbf{A}_{p^{\prime} / 2}}=\left[w^{2+\varepsilon}\right]_{\mathbf{A}_{1+\varepsilon}}^{\frac{1}{\varepsilon}} \leq\left[w^{2+\varepsilon}\right]_{\mathbf{A}_{1}}^{\frac{1}{\varepsilon}}$. By Theorem 1.2 there exists $\delta_{4}=\delta_{4}\left(N, \Lambda_{1}, \Lambda_{2}, \varepsilon,\left[w^{2+\varepsilon}\right]_{\mathbf{A}_{1}}\right) \in(0,1)$ such that if $\Omega$ is $\left(\delta_{4}, R_{0}\right)$-flat and $[A]_{s_{0}}^{R_{0}} \leq \delta_{4}$ for some $R_{0}>0$ there holds

$$
\left\|\left|\nabla \varphi \left\|_{L_{\bar{w}}^{p^{\prime}}\left(\Omega_{T}\right)} \leq c_{4}|\|G\||_{L_{\bar{w}}^{p^{\prime}}\left(\Omega_{T}\right)} \forall G \in C^{\infty}\left(\bar{\Omega}_{T}, \mathbb{R}^{N}\right),\right.\right.\right.
$$

where $\varphi$ is a unique solution to problem (3.15) and $c_{4}=c_{4}\left(N, \Lambda_{1}, \Lambda_{2}, \varepsilon,\left[w^{2+\varepsilon}\right]_{\mathbf{A}_{1}}\right)$. Using $\int_{\Omega_{T}} \nabla u G d x d t=\int_{\Omega_{T}} \nabla \varphi F$, Hölder inequality and (3.16) we find

$$
\left|\int_{\Omega_{T}} \nabla u G d x d t\right| \leq c_{4}|||F|||_{L_{w}^{p}\left(\Omega_{T}\right)}|||G|||_{L_{\frac{p^{\prime}}{p^{\prime}}\left(\Omega_{T}\right)}} \forall G \in C^{\infty}\left(\bar{\Omega}_{T}, \mathbb{R}^{N}\right) .
$$


Thus, we obtain

$$
\|\left.|| \nabla u||\right|_{L_{w}^{p}\left(\Omega_{T}\right)} \leq c_{4}|||F|||_{L_{w}^{p}\left(\Omega_{T}\right)} .
$$

On the other hand, by statement a there exist $\delta_{5}=\delta_{5}\left(N, \Lambda_{1}, \Lambda_{2},\left[w^{2+\varepsilon}\right]_{\mathbf{A}_{1}}\right) \in(0,1)$ such that if $\Omega$ is $\left(\delta_{5}, R_{0}\right)$-flat and $[A]_{s_{0}}^{R_{0}} \leq \delta_{5}$ for some $R_{0}>0$ there holds

$$
\|\left.|\nabla u|\right|_{L_{w}^{3}\left(\Omega_{T}\right)} \leq c_{5}|||F|||_{L_{w}^{3}\left(\Omega_{T}\right)} .
$$

for some $c_{5}=c_{5}\left(N, \Lambda_{1}, \Lambda_{2}, \varepsilon,\left[w^{2+\varepsilon}\right]_{\mathbf{A}_{1}}\right)$. We now denote map $\mathcal{J}:\left(L_{w}^{p}\left(\Omega_{T}\right)\right)^{N} \rightarrow$ $L_{w}^{p}\left(\Omega_{T}\right)$ by $\mathcal{J}(f):=|\nabla v|$ for any $f \in\left(L_{w}^{p}\left(\Omega_{T}\right)\right)^{N}$ where $v$ is the unique weak solution of problem (1.8) with $\mu \equiv 0, \sigma \equiv 0$ and $F=f$. We see that $\mathcal{J}$ is a sublinear operator and

$$
\left\|\mathcal{J}\left(f_{1}\right)\right\|_{L_{w}^{3}\left(\Omega_{T}\right)} \leq c_{5}||\left|f_{1}\right| \|_{L_{w}^{3}\left(\Omega_{T}\right)} \quad \forall f_{1} \in\left(L_{w}^{3}\left(\Omega_{T}\right)\right)^{N}
$$

and

$$
\left\|\mathcal{J}\left(f_{2}\right)\right\|_{L_{w}^{p}\left(\Omega_{T}\right)} \leq c_{4}||\left|f_{2}\right| \|_{L_{w}^{p}\left(\Omega_{T}\right)} \quad \forall f_{2} \in\left(L_{w}^{p}\left(\Omega_{T}\right)\right)^{N}
$$

where $\Omega$ is $\left(\delta, R_{0}\right)$-Reifenberg flat and $[A]_{s_{0}}^{R_{0}} \leq \delta$ with $\delta=\min \left\{\delta_{4}, \delta_{5}\right\}$. Thank to the interpolation Theorem, see [7, Theorem 1.4.19] we get the statement $\mathbf{b}$. This completes the proof.

\section{Quasilinear Riccati type parabolic equations}

To prove Theorem 1.5 we need the following Lemma:

Lemma 4.1 Let $\gamma \geq 1$ and $H_{1}, H_{2}$ be measurable functions in $\mathbb{R}^{N}$. If

$$
\int_{\Omega_{T}}\left|H_{1}\right| w d x d t \leq C\left(\gamma,\left[w^{\gamma}\right]_{\mathbf{A}_{1}}\right) \int_{\Omega_{T}}\left|H_{2}\right| w d x d t \quad \forall w^{\gamma} \in \mathbf{A}_{1},
$$

then for any $p>\frac{(N+2)(\gamma-1)}{\gamma}$,

$$
\left[\left|H_{1}\right|\right]_{\mathrm{Cap}_{\mathcal{G}_{1}, p}} \leq C\left[\left|H_{2}\right|\right]_{\mathrm{Cap}_{\mathcal{G}_{1}, p}},
$$

where $C=C\left(N, p, \gamma, T_{0}\right)$, for measurable function $H$ in $\mathbb{R}^{N},[H]_{\text {Cap }_{\mathcal{G}_{1}, p}}$ is denoted by

$$
[|H|]_{\mathrm{Cap}_{\mathcal{G}_{1}, p}}=\sup \frac{\int_{K}|H| d x d t}{\operatorname{Cap}_{\mathcal{G}_{1}, p}(K)},
$$

the suprema being taken over all compact sets $K \subset \mathbb{R}^{N+1}$.

Its proof can be found in [19, Proof of Propostion 4.24]. Using this Lemma we obtain

Theorem 4.2 Suppose that $A$ is linear. Let $F \in L^{1}\left(\Omega_{T}, \mathbb{R}^{N}\right), \mu \in \mathfrak{M}_{b}\left(\Omega_{T}\right), \sigma \in \mathfrak{M}_{b}(\Omega)$, set $\omega=|\mu|+|\sigma| \otimes \delta_{\{t=0\}}$. Let $s_{0}$ be in Theorem 1.1.

(a) For any $q>1$ and $\mathcal{M}_{1}[\omega], \mathcal{M}\left(|F|^{2}\right)^{1 / 2} \in L^{q}\left(\Omega_{T}\right)$ we find $\delta=\delta\left(N, \Lambda_{1}, \Lambda_{2}, q\right) \in(0,1)$ such that if $\Omega$ is a $\left(\delta, R_{0}\right)$-Reifenberg flat domain and $[A]_{s_{0}}^{R_{0}} \leq \delta$ for some $R_{0}>0$ then there exists a unique weak solution $u \in L^{q}\left(0, T, W_{0}^{1, q}\right)$ of (1.8) and there holds

$$
\left[|\nabla u|^{q} \chi_{\Omega_{T}}\right]_{\mathrm{Cap}_{\mathcal{G}_{1}, q^{\prime}}} \leq C_{1}\left[\left(\mathcal{M}\left(|F|^{2}\right)\right)^{q / 2} \chi_{\Omega_{T}}\right]_{\mathrm{Cap}_{\mathcal{G}_{1}, q^{\prime}}}+C_{1}[\omega]_{\mathrm{Cap}_{\mathcal{G}_{1}, q^{\prime}}}^{q}
$$

where $C_{1}=C_{1}\left(N, \Lambda_{1}, \Lambda_{2}, q, T_{0} / R_{0}, T_{0}\right)$. 
(b) For any $1<q<\frac{N+2}{N}$ and $\mathcal{M}_{1}[\omega],|F| \in L^{q}\left(\Omega_{T}\right)$ we find $\delta=\delta\left(N, \Lambda_{1}, \Lambda_{2}, q\right) \in(0,1)$ such that if $\Omega$ is a $\left(\delta, R_{0}\right)$-flat domain and $[A]_{s_{0}}^{R_{0}} \leq \delta$ for some $R_{0}>0$ then there exists a unique weak solution $u \in L^{q}\left(0, T, W_{0}^{1, q}(\Omega)\right)$ of (1.8) and there holds

$$
\left[|\nabla u|^{q} \chi_{\Omega_{T}}\right]_{\operatorname{Cap}_{\mathcal{G}_{1}, q^{\prime}}} \leq C_{2}\left[|F|^{q} \chi_{\Omega_{T}}\right]_{\operatorname{Cap}_{\mathcal{G}_{1}, q^{\prime}}}+C_{2}[\omega]_{\operatorname{Cap}_{\mathcal{G}_{1}, q^{\prime}}}^{q}
$$

where $C_{2}=C_{2}\left(N, \Lambda_{1}, \Lambda_{2}, q, T_{0} / R_{0}, T_{0}\right)$.

Proof of Theorem 3.1 We have

$$
\left[\left(\mathcal{M}_{1}[\omega]\right)^{q} \chi_{\Omega_{T}}\right]_{\mathrm{Cap}_{\mathcal{G}_{1}, q^{\prime}}} \leq c[\omega]_{\mathrm{Cap}_{\mathcal{G}_{1}, q^{\prime}}}^{q},
$$

where $c=c\left(N, q, T_{0}\right)$, see [19, Corollary 4.39]. Therefore, thanks to Theorems 1.1, 1.3 and Lemma 4.1 we get the results.

Proof of Theorem 1.5 By Theorem 4.2, there exists $\delta=\delta\left(N, \Lambda_{1}, \Lambda_{2}, q\right) \in(0,1)$ such that $\Omega$ is $\left(\delta, R_{0}\right)$ - Reifenberg flat domain and $[\mathcal{A}]_{s_{0}}^{R_{0}} \leq \delta$ for some $R_{0}$ and a sequence $\left\{u_{n}\right\}_{n}$ obtained by induction of the weak solutions of

$$
\left\{\begin{array}{l}
\left(u_{1}\right)_{t}-\operatorname{div}\left(A\left(x, t, \nabla u_{1}\right)\right)=\operatorname{div}(F)+\mu \text { in } \Omega_{T}, \\
u_{1}=0 \text { on } \partial \Omega \times(0, T) \\
u_{1}(0)=\sigma \text { in } \Omega
\end{array}\right.
$$

and

$$
\left\{\begin{array}{l}
\left(u_{n+1}\right)_{t}-\operatorname{div}\left(A\left(x, t, \nabla u_{n+1}\right)\right)=\left|\nabla u_{n}\right|^{q}+\operatorname{div}(F)+\mu \text { in } \Omega_{T} \\
u_{n+1}=0 \text { on } \partial \Omega \times(0, T) \\
u_{n+1}(0)=\sigma \quad \text { in } \Omega
\end{array}\right.
$$

for any which satisfy

$$
\left[\left|\nabla u_{n+1}\right|^{q} \chi \Omega_{T}\right]_{\operatorname{Cap}_{\mathcal{G}_{1}, q^{\prime}}} \leq c\left[H_{q}\right]_{\operatorname{Cap}_{\mathcal{G}_{1}, q^{\prime}}}+c\left[\left|\nabla u_{n}\right|^{q} \chi_{\Omega_{T}}\right]_{\operatorname{Cap}_{\mathcal{G}_{1}, q^{\prime}}}^{q}+c[\omega]_{\mathrm{Cap}_{\mathcal{G}_{1}, q^{\prime}}}^{q} \quad \forall n \geq 0
$$

where $u_{0} \equiv 0$ and the constant $c$ depends only on $N, \Lambda_{1}, \Lambda_{2}, q$ and $T_{0} / R_{0}, T_{0}$. Since, $u_{n+1}-u_{n}$ is the unique weak solution of

$$
\left\{\begin{array}{l}
u_{t}-\operatorname{div}(A(x, t, \nabla u))=\left|\nabla u_{n}\right|^{q}-\left|\nabla u_{n-1}\right|^{q} \text { in } \Omega_{T}, \\
u=0 \text { on } \partial \Omega \times(0, T), \\
u(0)=0 \text { in } \Omega
\end{array}\right.
$$

we have

$$
\left[\left|\nabla u_{n+1}-\nabla u_{n}\right|^{q} \chi_{\Omega_{T}}\right]_{\operatorname{Cap}_{\mathcal{G}_{1}, q^{\prime}}} \leq c\left[\left.|| \nabla u_{n}\right|^{q}-\left|\nabla u_{n-1}\right|^{q} \mid \chi_{\Omega_{T}}\right]_{\mathrm{Cap}_{\mathcal{G}_{1}, q^{\prime}}^{q}} \quad \forall n \geq 0 .
$$

If

$$
\left[H_{q}\right]_{\operatorname{Cap}_{\mathcal{G}_{1}, q^{\prime}}}+[\omega]_{\operatorname{Cap}_{\mathcal{G}_{1}, q^{\prime}}}^{q} \leq \frac{q-1}{(c q)^{q^{\prime}}},
$$

then from (4.3) we can show that

$$
\left[\left|\nabla u_{n}\right|^{q} \chi_{\Omega_{T}}\right]_{\operatorname{Cap}_{\mathcal{G}_{1}, q^{\prime}}} \leq c q^{\prime}\left(\left[H_{q}\right]_{\operatorname{Cap}_{\mathcal{G}_{1}, q^{\prime}}}+[\omega]_{\operatorname{Cap}_{\mathcal{G}_{1}, q^{\prime}}}^{q}\right) \quad \forall n \geq 1 .
$$


Using Hölder inequality and (4.5) yield

$$
\begin{aligned}
& {\left[\left|\nabla u_{n+1}-\nabla u_{n}\right|^{q} \chi_{\Omega_{T}}\right]_{\operatorname{Cap}_{\mathcal{G}_{1}, q^{\prime}}} \leq c q\left[|| \nabla u_{n}-\nabla u_{n-1} \mid\left(\left|\nabla u_{n}\right|^{q-1}+\left|\nabla u_{n-1}\right|^{q-1}\right) \chi_{\Omega_{T}}\right]_{\mathrm{Cap}_{\mathcal{G}_{1}, q^{\prime}}}^{q}} \\
& \leq c q\left[\left|\nabla u_{n}-\nabla u_{n-1}\right|^{q} \chi_{\Omega_{T}}\right]_{\operatorname{Cap}_{\mathcal{G}_{1}, q^{\prime}}} \\
& \times\left[\left(\left|\nabla u_{n}\right|^{q-1}+\left|\nabla u_{n-1}\right|^{q-1}\right)^{q^{\prime}} \chi_{\Omega_{T}}\right]_{\mathrm{Cap}_{\mathcal{G}_{1}, q^{\prime}}}^{q-1} \\
& \leq c q 2^{q^{\prime}-1}\left[\left|\nabla u_{n}-\nabla u_{n-1}\right|^{q} \chi_{\Omega_{T}}\right]_{\mathrm{Cap}_{\mathcal{G}_{1}, q^{\prime}}} \\
& \times\left(\left[\left|\nabla u_{n}\right|^{q} \chi_{\Omega_{T}}\right]_{\operatorname{Cap}_{\mathcal{G}_{1}, q^{\prime}}^{q-1}}^{-1}+\left[\left|\nabla u_{n-1}\right|^{q} \chi_{\Omega_{T}}\right]_{\operatorname{Cap}_{\mathcal{G}_{1}, q^{\prime}}}^{q-1}\right) .
\end{aligned}
$$

Hence, by (4.6)-(4.7) we find

$$
\begin{aligned}
& {\left[\left|\nabla u_{n+1}-\nabla u_{n}\right|^{q} \chi_{\Omega_{T}}\right]_{\operatorname{Cap}_{\mathcal{G}_{1}, q^{\prime}}}} \\
& \quad \leq c q 2^{q^{\prime}}\left(c q^{\prime}\right)^{q-1}\left(\left[H_{q}\right]_{\operatorname{Cap}_{\mathcal{G}_{1}, q^{\prime}}}+[\omega]_{\operatorname{Cap}_{\mathcal{G}_{1}, q^{\prime}}}^{q}\right)^{q-1}\left[\left|\nabla u_{n}-\nabla u_{n-1}\right|^{q} \chi_{\Omega_{T}}\right]_{\operatorname{Cap}_{\mathcal{G}_{1}, q^{\prime}}} \\
& \quad \leq \frac{1}{2}\left[\left|\nabla u_{n}-\nabla u_{n-1}\right|^{q} \chi_{\Omega_{T}}\right]_{\operatorname{Cap}_{\mathcal{G}_{1}, q^{\prime}}},
\end{aligned}
$$

provided that

$$
\left[H_{q}\right]_{\operatorname{Cap}_{\mathcal{G}_{1}, q^{\prime}}}+[\omega]_{\operatorname{Cap}_{\mathcal{G}_{1}, q^{\prime}}}^{q} \leq \min \left\{\left(c q^{\prime} 2^{q^{\prime}+1}\left(c q^{\prime}\right)^{q-1}\right)^{-\frac{1}{q-1}}, \frac{q-1}{(c q)^{q^{\prime}}}\right\} .
$$

Hence, if (4.8) holds, $u_{n}$ converges to $u=u_{1}+\sum_{n=1}^{\infty}\left(u_{n+1}-u_{n}\right)$ in $L^{q}\left(0, T, W_{0}^{1, q}(\Omega)\right)$ satisfying

$$
\left[|\nabla u|^{q} \chi_{\Omega_{T}}\right]_{\mathrm{Cap}_{\mathcal{G}_{1}, q^{\prime}}} \leq c q^{\prime}\left(\left[H_{q}\right]_{\mathrm{Cap}_{\mathcal{G}_{1}, q^{\prime}}}+[\omega]_{\operatorname{Cap}_{\mathcal{G}_{1}, q^{\prime}}}^{q}\right) .
$$

Obviously, $u$ is a weak solution of problem (1.15). This completes the proof.

Acknowledgments The author wishes to express his deep gratitude to Professor Laurent Véron and Professor Marie-Françoise Bidaut-Véron for encouraging, taking care and giving many useful comments during the preparation of the paper. Besides, the author would like to thank the anonymous referee for giving many remarks about the papers $[5,8,16,17]$.

\section{References}

1. Bagby, R.J.: Lebesgue spaces of parabolic potentials. Ill. J. Math. 15, 610-634 (1971)

2. Byun, S.S., Wang, L.: Parabolic equations with BMO nonlinearity in Reifenberg domains. J. Reine Angew. Math. 615, 1-24 (2008)

3. Byun, S.S., Wang, L.: Parabolic equations in Reifenberg domains. Arch. Ration. Mech. Anal. 176(2), 271-301 (2005)

4. Byun, S.S., Ok, J., Ryu, S.: Global gradient estimates for general nonlinear parabolic equations in nonsmooth domains. J. Differ. Equ. 254(11), 4290-4326 (2013)

5. Caffarelli, L., Peral, I.: On $W^{1, p}$ estimates for elliptic equations in divergence form. Commun. Pure Appl. Math. 51, 1-21 (1998)

6. Duzaar, F., Mingione, G.: Gradient estimate via non-linear potentials. Am. J. Math. 133(4), 1093-1149 (2011)

7. Grafakos, L.: Classical and Modern Fourier Analysis, pp. xii+931. Pearson Education, Inc., Upper Saddle River (2004) 
8. Iwaniec, T.: Projections onto gradient fields and L p-estimates for degenerated elliptic operators. Stud. Math. 75, 293-312 (1983)

9. Kenig, C., Toro, T.: Free boundary regularity for harmonic measures and the Poisson kernel. Ann. Math. 150, 367-454 (1999)

10. Kenig, C., Toro, T.: Poisson kernel characterization of Reifenberg at chord arc domains. Ann. Sci. École Norm. Sup. 36, 323-401 (2003)

11. Lieberman, G.M.: Boundary regularity for solutions of degenerate parabolic equations. Nonlinear Anal. 14(6), 501-524 (1990)

12. Lieberman, G.M.: Boundary and initial regularity for solutions of degenerate parabolic equations. Nonlinear Anal. 20(5), 551-569 (1993)

13. Mengesha, T., Phuc, N.C.: Global estimates for quasilinear elliptic equations on Reifenberg flat domains. Arch. Ration. Mech. Anal. 203, 189-216 (2012)

14. Mengesha, T., Phuc, N.C.: Quasilinear Riccati type equations with distributional data in Morrey space framework. https://www.mittag-leffler.se/preprints/files/IML-1314f-23.pdf

15. Mengesha, T., Phuc, N.C.: Weighted and regularity estimates for nonlinear equations on Reifenberg flat domains. J. Differ. Equ. 250(5), 1485-2507 (2011)

16. Mingione, G.: The Calderón-Zygmund theory for elliptic problems with measure data. Ann. Scu. Norm. Sup. Pisa Cl. Sci. 6(5), 195-261 (2007)

17. Mingione, G.: Gradient estimates below the duality exponent. Math. Ann. 346(3), 571-627 (2010)

18. Naumann, J., Wolf, J.: Interior Integral Estimates on Weak Solutions of Nonlinear Parabolic Systems. Humboldt Universitet, Bonn (1994)

19. Nguyen, Q.-H.: Potential estimates and quasilinear parabolic equations with measure data. https://hal. inria.fr/hal-00989464v2/document

20. Reinfenberg, E.: Solutions of the plateau problem for m-dimensional surfaces of varying topological type. Acta Math. 104, 1-92 (1960)

21. Stein, E.M.: Singular Integrals and Differentiability Properties of Functions, Princeton Mathematical Series, 30. Princeton University Press, Princeton (1970)

22. Stein, E.M.: Harmonic Analysis: Real-Variable Methods, Orthogonality, and Oscillatory Integral, 43. Princeton University Press, Princeton (1993)

23. Toro, T.: Doubling and flatness: geometry of measures. Not. Am. Math. Soc. 44, 1087-1094 (1997)

24. Tureson, B.O.: Nonlinear Potential Theory and Weighted Sobolev Spaces. Lecture Notes in Mathematics, vol. 1736. Springer, Berlin (2000) 